\title{
THREE EMBEDDED TECHNIQUES FOR FINITE ELEMENT HEAT FLOW PROBLEM WITH EMBEDDED DISCONTINUITIES
}

\author{
M.Davari ${ }^{1}$, R.Rossi $^{2}$ and P.Dadvand ${ }^{2}$ \\ ${ }^{1}$ Department of Strength of Materials and Structural Engineering, Polytechnic University of \\ Catalonia, Barcelona, Spain \\ ${ }^{2}$ International Center for Numerical Methods in Engineering (CIMNE), Department of Strength of \\ Materials and Structural Engineering, Polytechnic University of Catalonia, Barcelona, Spain
}

\begin{abstract}
The present paper explores the solution of a heat conduction problem considering discontinuities embedded within the mesh and aligned at arbitrary angles with respect to the mesh edges.

Three alternative approaches are proposed as solutions to the problem. The difference between these approaches compared to alternatives, such as the eXtended Finite Element Method (X-FEM), is that the current proposal attempts to preserve the global matrix graph in order to improve performance. The first two alternatives comprise an enrichment of the Finite Element (FE) space obtained through the addition of some new local degrees of freedom to allow capturing discontinuities within the element. The new degrees of freedom are statically condensed prior to assembly, so that the graph of the final system is not changed. The third approach is based on the use of modified FE-shape functions that substitute the standard ones on the cut elements. The imposition of both Neumann and Dirichlet boundary conditions is considered at the embedded interface. The results of all the proposed methods are then compared with a reference solution obtained using the standard FE on a mesh containing the actual discontinuity.
\end{abstract}

\section{INTRODUCTION}

This paper focuses on the solution of a conduction problem on a domain containing a gap at a prescribed position in space. The salient feature of the work is that, instead of modifying the geometry of the domain so that the gap is modelled as a physical boundary, we embed the geometrical description of the structure in a mesh that is continuous across the gap.

The introduction of such a gap implies that the physical solution suffers from a discontinuity, potentially both in the primary variable (the temperature) and in its gradients. This feature makes it difficult to approximate the solution with a standard, continuous FE approach, unless the mesh is body-fitted to the gap geometry.

Perhaps the most well-known approach to the treatment of discontinuities within the element is eXtended Finite Element Method (X-FEM) [1-8]. The X-FEM is a numerical technique where enrichment functions are added to the FE approximation space so as to provide it with the ability to reproduce specific features of the solution. This enrichment is normally done only at the region near the discontinuities such as cracks, holes, and similar material interfaces. In X-FEM the numerical model comprises two types of elements at the region near the discontinuity: fully enriched elements that are cut by the discontinuity and partially enriched elements (blending elements) that are not cut by the discontinuity but have one or more enriched nodes (see Figure 1). Two disadvantages of standard X-FEM specially for moving discontinuities may be mentioned: 
1. The graph of the final system is changed due to the need for adding global degrees of freedom into the FE approximation space.

2. The enrichment functions should be considered not only for the elements that contain the discontinuity but also for those elements adjacent to them.

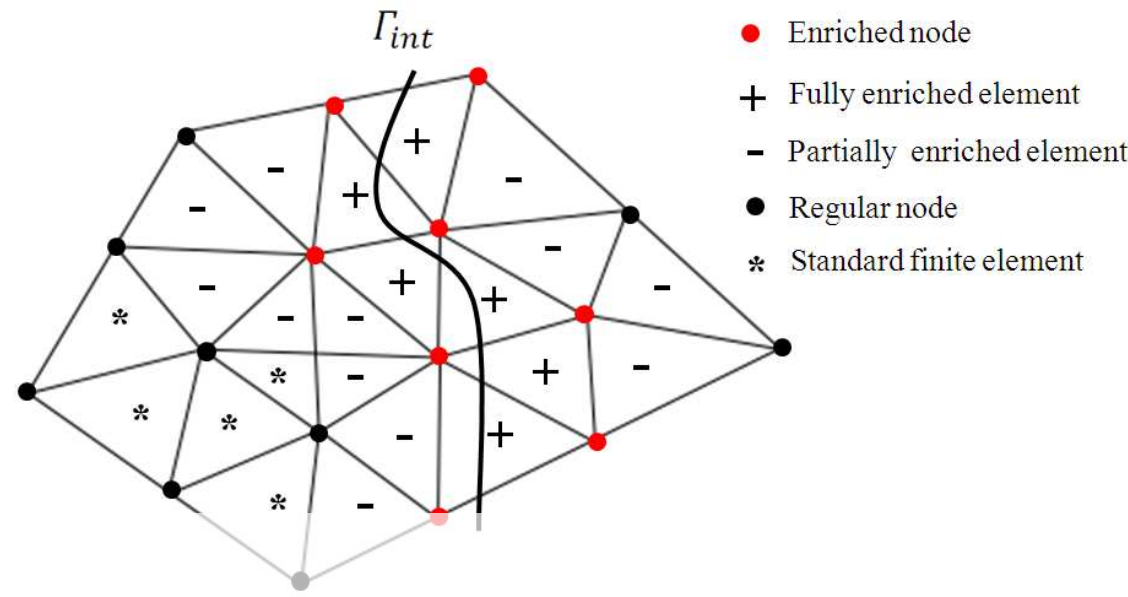

Figure 1: Enriched nodes and elements for standard X-FEM

Based on these difficulties, we explore the use of three distinct methods capable of capturing discontinuities within the element but without the issue of blending elements.

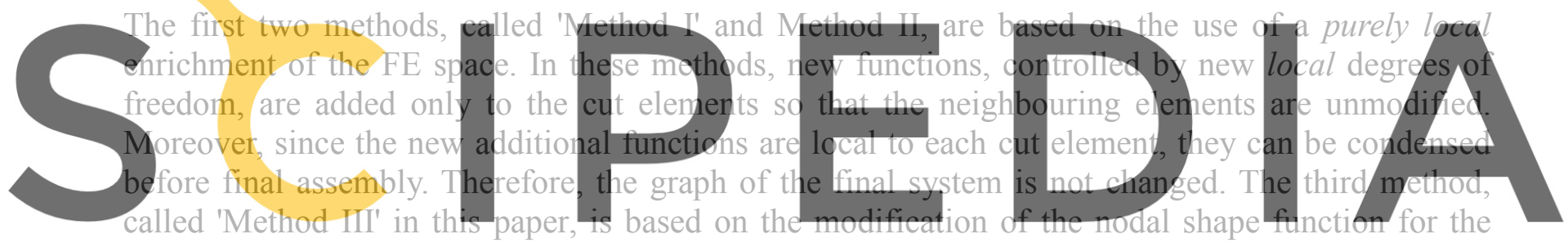
sole elements, which are cut by discontinuities.

Register for free at https//www.scipedia.com to download the version without the watermark Independent of the specific choice made in the definition of the improved approximation scheme, the new shape functions shall guarantee the regularity requirements needed for the solution of the problem of interest. For the conduction problem, this requires the shape functions to be in $H^{1}$, except across the embedded gap. This is verified by the replacement shape functions we consider as part of Method III, not through the enrichments we explore in Methods I and II. The practical implication of the first two methods is a 'variational crime' that invalidates the available convergence proof. In the present work we show heuristically how these methods appear to work reliably despite this lack of guarantee.

Thus, the paper is structured as follows: We begin by writing the governing equations for the heat conduction problem. In Sections 3, 4, and 5 we describe our proposed methods. We discuss the imposition of Neumann and Dirichlet conditions on the embedded boundary in Section 6. Section 7 concludes the paper with the discussion of benchmark problems.

\section{GOVERNING EQUATIONS FOR HEAT CONDUCTION}

Consider the domain $\Omega \subset \mathbb{R}^{2}$ as shown in Figure 2 with an immersed discontinuity $\Gamma_{\text {int }}$. The equation for transient heat transfer by conduction in the absence of energy sources is: 


$$
\rho c_{p} \frac{\partial \boldsymbol{T}}{\partial t}-\nabla \cdot(k \nabla \boldsymbol{T})=0 \text { in } \Omega
$$

where $\boldsymbol{T}$ is the temperature, $t$ is the time, $\rho$ is the material's density, $c_{p}$ its specific heat, and $k$ is the thermal conductivity. For the heat equation, various types of boundary conditions can be considered:

$$
\begin{aligned}
\boldsymbol{T} & =\boldsymbol{T}_{\boldsymbol{c}} & & \text { on } \Gamma_{c} \\
\boldsymbol{T} & =\boldsymbol{T}_{\text {int }} & & \text { on } \Gamma_{\text {int }} \\
k \nabla \boldsymbol{T} . n & =\boldsymbol{q}_{\text {int }} & & \text { on } \Gamma_{\text {int }}
\end{aligned}
$$

where $\boldsymbol{T}_{\boldsymbol{c}}$ represents the wall temperature imposed on a portion of the boundary $\boldsymbol{\Gamma}_{\boldsymbol{c}}, \boldsymbol{T}_{\boldsymbol{i n t}}$ is the prescribed temperature imposed on $\boldsymbol{\Gamma}_{\text {int }}$ (Dirichlet type boundary condition ), and $\boldsymbol{q}_{\text {int }}$ is a prescribed heat flux imposed on $\boldsymbol{\Gamma}_{\boldsymbol{i n t}}$ (Neumann type boundary condition). The Galerkin FE formulation is obtained by multiplying Eq. (1) by an appropriate test function $w$ and by integrating over the computational domain [9].
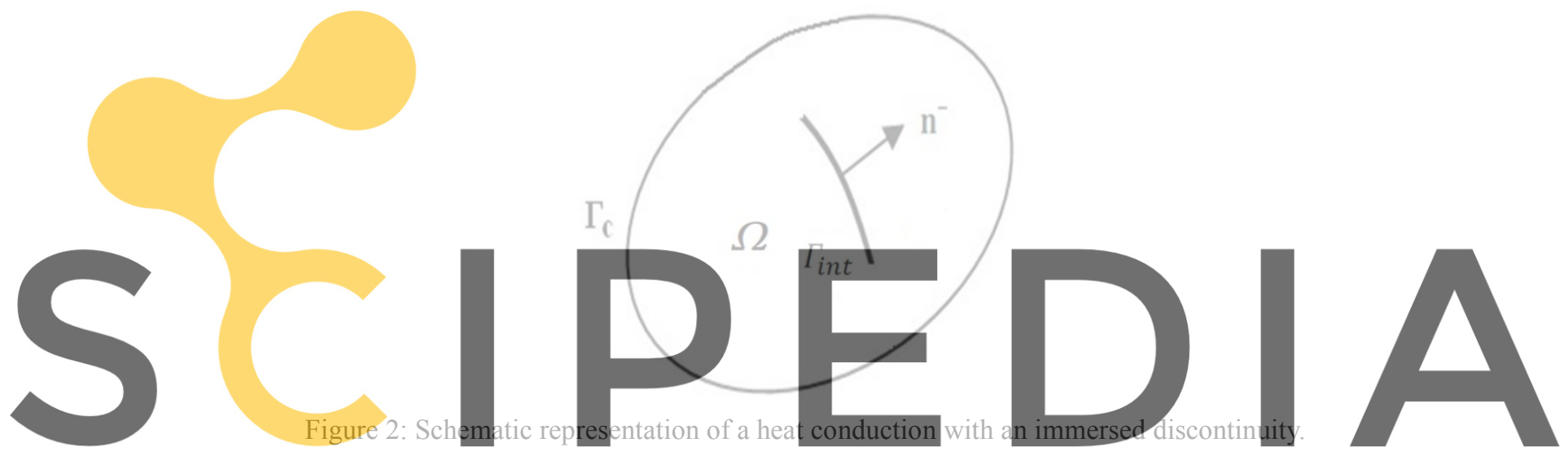

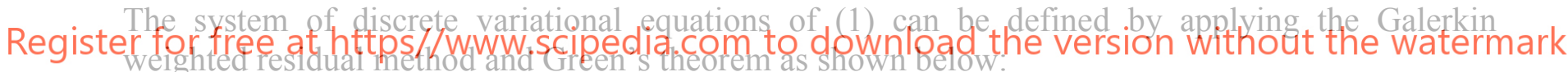

where $w$ is test functions and

$$
a\left(\frac{\partial \boldsymbol{T}}{\partial t}, w\right)+b(\boldsymbol{T}, w)=l(w)
$$

$$
\begin{aligned}
a\left(\frac{\partial \boldsymbol{T}}{\partial t}, w\right) & =\left(\frac{\rho C_{P} \partial \boldsymbol{T}}{\partial t}, w\right) \\
b(\boldsymbol{T}, w) & =(k \nabla \boldsymbol{T}, \nabla w) \\
l(w) & =\left(\boldsymbol{q}_{\boldsymbol{n}}, w\right)
\end{aligned}
$$

To determine the FE field, the solution domain $\Omega$ is divided into a set of finite number of triangle elements $\Omega^{h}$. Hence, the temperature within the element is interpolated from the nodal values and can be written as:

$$
\boldsymbol{T}^{h}(x)=\sum_{i=1}^{3} N_{i}(x) \boldsymbol{T}_{i}
$$

where $N_{i}$ is the shape function and $\boldsymbol{T}_{i}$ is the nodal temperature of the node $\mathrm{i}^{\text {th }}$ of the element. By 
substituting (9) in the weak form (5), we obtain the following matrix form:

$$
\boldsymbol{C} \frac{\partial \boldsymbol{T}}{\partial t}-\boldsymbol{K} \boldsymbol{T}=\boldsymbol{Q}+\boldsymbol{S}
$$

where $\boldsymbol{T}$ is the vector of nodal unknown temperatures, $\boldsymbol{C}$ is the capacitance matrix, $\boldsymbol{K}$ is the stiffness or conductivity matrix, $\boldsymbol{Q}$ is the Neumann boundary or the flux term at the boundaries of the triangle element, and $\boldsymbol{S}$ is the external flux term at the immersed boundary and

$$
\begin{array}{rlrl}
\boldsymbol{C}_{i j} & =\int_{\Omega^{h}} \rho c_{p} N_{i} N_{j} d \Omega & i, j=1,3 \\
\boldsymbol{K}_{i j}=-\int_{\Omega^{h}} k \nabla N_{i} \cdot \nabla N_{j} d \Omega & i, j=1,3 \\
\boldsymbol{O}_{i}=\int_{\Gamma_{h}} \boldsymbol{q}_{n} N_{i} d \Gamma & i=1,3 \\
S_{i}=\int_{\Gamma_{\text {int }}} q_{n} N_{i} d \Gamma_{\text {int }} & i=1,3 \\
F_{i}=\int_{\Omega^{h}} f N_{i} d \Omega & i=1,3
\end{array}
$$

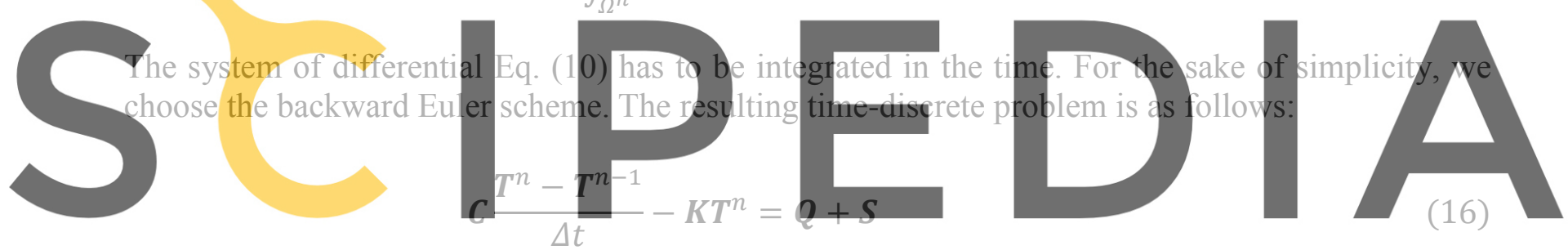

Register for free at https//www.scipedia.com to download the version without the watermark 3. METHOD I (ENRICHMENT FINITE ELEMENT METHOD)

As already mentioned, we seek to propose three distinct techniques to embed the discontinuity of interest in the search space. In this section, we introduce a purely local enrichment of the FE space. Four new degrees of freedom are added locally to the sole elements intersected by the embedded gap, with the objective of capturing discontinuities (kinks and jumps) in the solution. The enriched basis is formed by the combination of the nodal shape functions associated with the mesh (standard FE part) and discontinuous shape functions associated with additional degrees of freedom (enrichment parts). For the rest of the elements, only the standard FE interpolation will be applied.

As mentioned in [8], one possible way to construct a suitable enriched space ( $H^{1}$ everywhere apart across the gap) is by adding some new degrees of freedom to the cut elements with enriched functions. For example, if the field is scalar, a three-node triangular element that is not cut by the $\Gamma_{\text {int }}$ has one degree of freedom per node. However, the degree of freedom would be two if the element were cut (one more due to using the enriched functions to capture discontinuity within the element). Thus, the element adjacent to a cut element would have one or more enriched nodes even though the element may not contain $\Gamma_{\text {int }}$. In other words, not only the elements that are cut by $\Gamma_{\text {int }}$ but also their immediate neighbours are enriched [10].

The key differentiator of our method with respect to what mentioned in [8] is that the enriched functions are added to the cut elements using new local degrees of freedom, which do not depend 
on any of the neighbouring elements. The key idea we exploit is that since the new degrees of freedom are purely local, they can be statically condensed at the elementary level before the final assembly. Therefore, the size and the graph of the final linear system to be solved are the same as in the standard case.

While the locality of this approach is very appealing from a computational point of view, it is clear that, as the enrichment functions are not zero on the element boundaries, discontinuous solutions can be constructed not only across the boundary but also within one enriched element and the neighbour. This implies that $C^{0}$ continuity is violated across the edges intersected by the interface (Figure 3). Hence, a variational crime is produced if the proposed shape functions are employed. We later show how this 'crime' seems to pay off, since a satisfactory behaviour is found in all of the test examples we considered, while a very efficient implementation becomes possible due to the strict locality of the proposed approach.

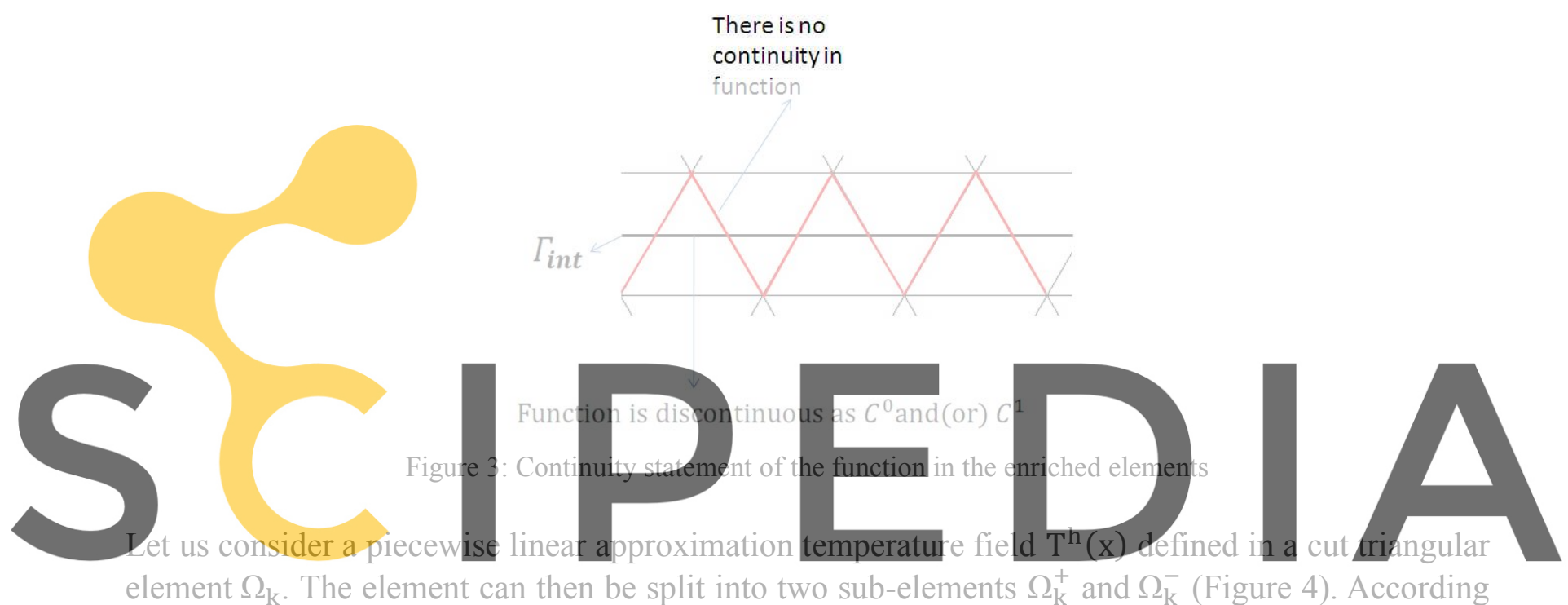

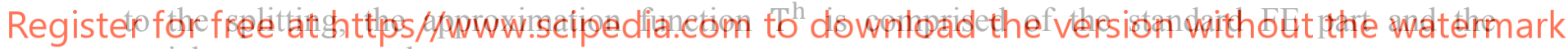
enrichment parts such as

$$
\boldsymbol{T}^{h}(x)=\sum_{i=1}^{3} N_{i}(x) \boldsymbol{T}_{i}+\sum_{m=1}^{2} \widetilde{N}_{m}(x) \widetilde{\boldsymbol{T}}_{m}+\sum_{m=1}^{2} \widehat{N}_{m}(x) \widehat{\boldsymbol{T}}_{m}
$$

where on the right-hand side of the equation, the first term is the standard FE part, and the second and third terms are enrichment parts related to weak (kinks) and strong (jump) discontinuities respectively. The individual variables stand for

$T^{h}(x)$ : Approximated function

$N_{i}(x)$ : Standard FE function for node $\mathrm{i}$

$\boldsymbol{T}_{i} \quad$ : Standard temperature degrees of freedom for node $\mathrm{i}$

$\widetilde{N}_{m}(x)$ : Local enrichment functions for weak discontinuity part

$\widetilde{\boldsymbol{T}}_{m} \quad$ : Additional degrees of freedom, associated with weak discontinuity part

$\widehat{N}_{m}(x)$ : Local enrichment function for strong discontinuity part

$\widehat{\boldsymbol{T}}_{m} \quad$ : Additional degrees of freedom, associated with strong discontinuity part 


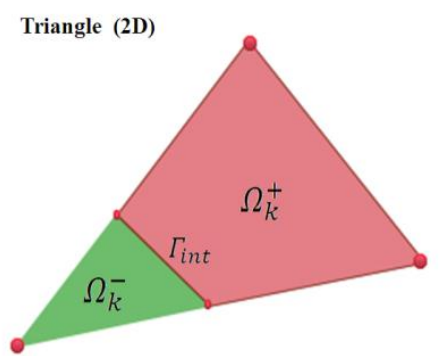

Figure 4. Typical element $\boldsymbol{\Omega}_{\boldsymbol{k}}$ and subelements $\boldsymbol{\Omega}_{\boldsymbol{k}}^{+}$and $\boldsymbol{\Omega}_{\boldsymbol{k}}^{-}$and interface $\Gamma_{\boldsymbol{i n t}}$.

Figure 5 shows the weak and strong discontinuous shape functions for typical triangular element.
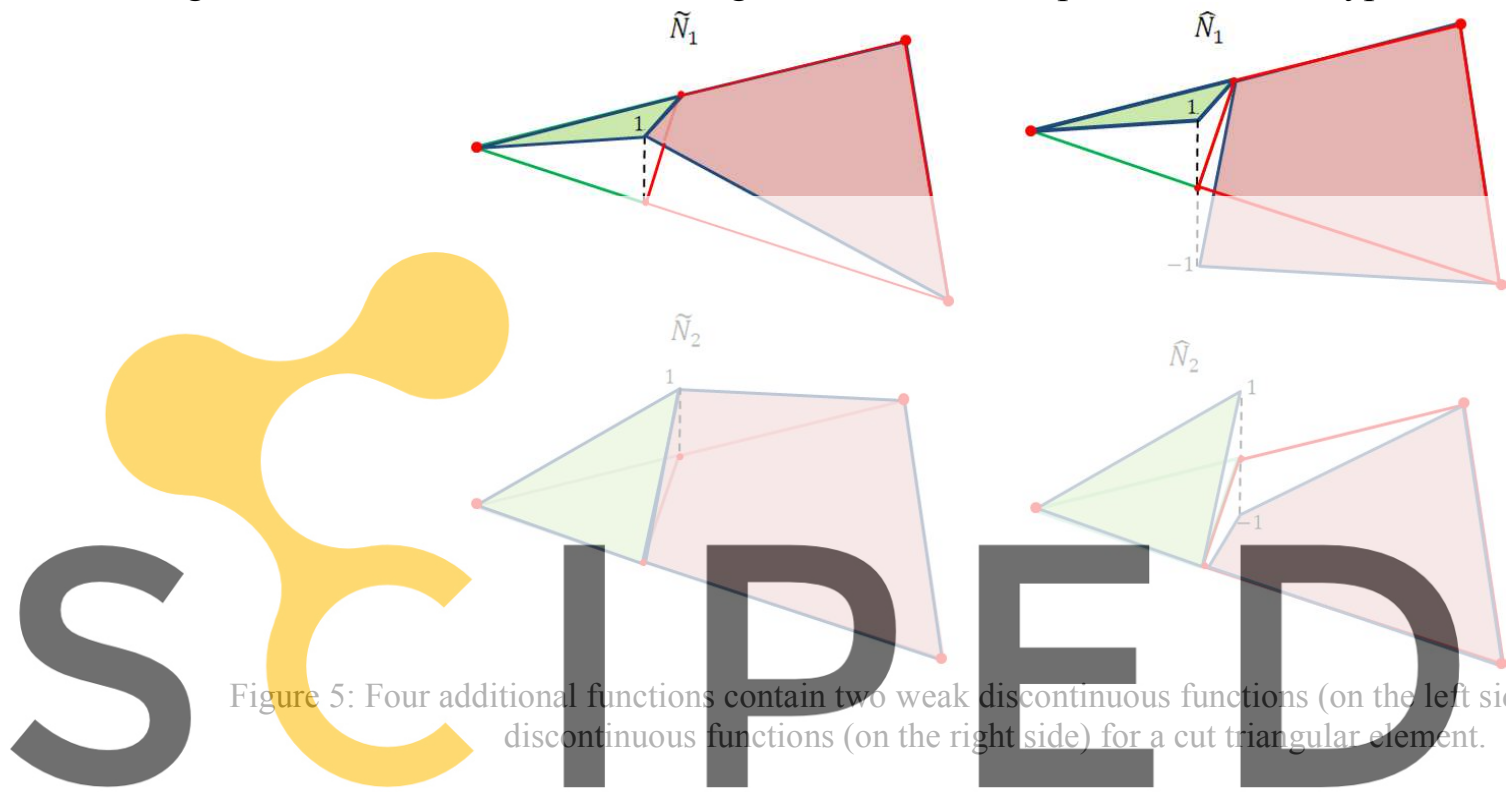

Figure 5: Four additional
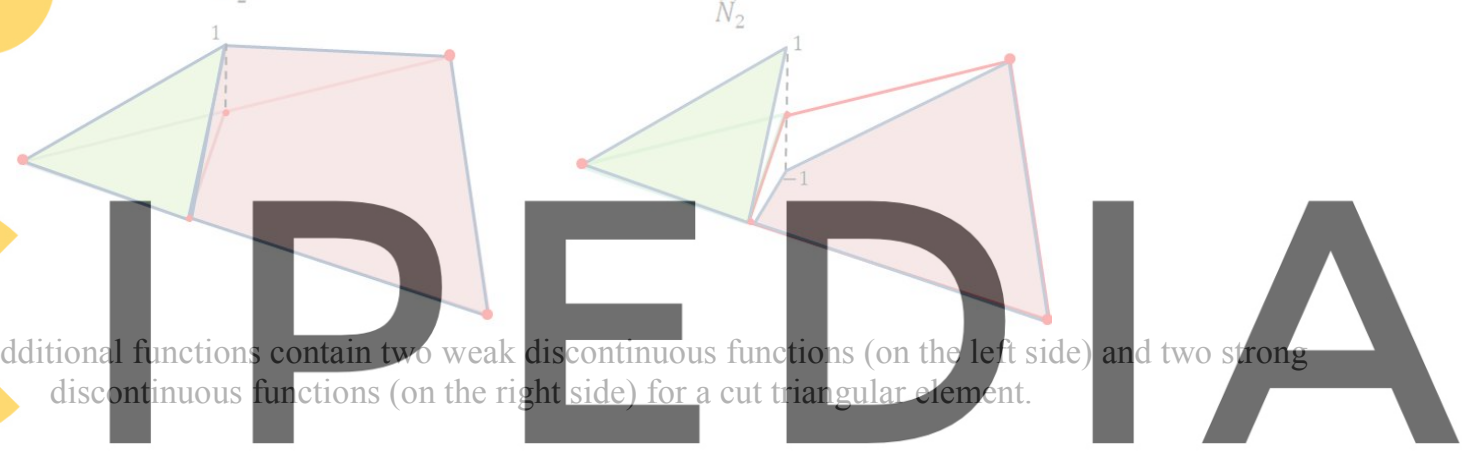

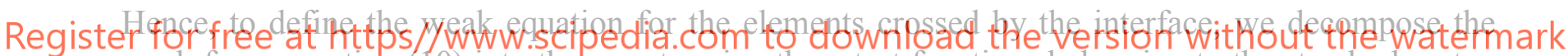
weak form equation (10) into three parts using three test functions belonging to the standard part $w$ and the enrichment parts (weak part $\widetilde{w}$ and strong part $\widehat{w}$ ), whereupon we substitute the equation (17) into it. This results in the following:

$\left(\boldsymbol{C}_{s s} \frac{\partial \boldsymbol{T}}{\partial t}+\boldsymbol{C}_{s e} \frac{\partial \widetilde{\boldsymbol{T}}}{\partial t}+\boldsymbol{C}_{s h} \frac{\partial \widehat{\boldsymbol{T}}}{\partial t}\right)-\left[\left(\boldsymbol{K}_{s s} \boldsymbol{T}+\boldsymbol{K}_{s e} \widetilde{\boldsymbol{T}}+\boldsymbol{K}_{s h} \widehat{\boldsymbol{T})}+\left(\boldsymbol{Q}_{s s} \boldsymbol{T}+\boldsymbol{Q}_{s e} \widetilde{\boldsymbol{T}}+\boldsymbol{Q}_{s h} \widehat{\boldsymbol{T}}\right)\right]=\boldsymbol{S}_{s}\right.$

$\left(\boldsymbol{C}_{e s} \frac{\partial \boldsymbol{T}}{\partial t}+\boldsymbol{C}_{e e} \frac{\partial \widetilde{\boldsymbol{T}}}{\partial t}+\boldsymbol{C}_{e h} \frac{\partial \widehat{\boldsymbol{T}}}{\partial t}\right)-\left[\left(\boldsymbol{K}_{e s} \boldsymbol{T}+\boldsymbol{K}_{e e} \widetilde{\boldsymbol{T}}+\boldsymbol{K}_{e h} \widehat{\boldsymbol{T}}\right)+\left(\boldsymbol{Q}_{e s} \boldsymbol{T}+\boldsymbol{Q}_{e e} \widetilde{\boldsymbol{T}}+\boldsymbol{Q}_{e h} \widehat{\boldsymbol{T}}\right)\right]=\boldsymbol{S}_{e}$

$\left(\boldsymbol{C}_{h s} \frac{\partial \boldsymbol{T}}{\partial t}+\boldsymbol{C}_{h e} \frac{\partial \widetilde{\boldsymbol{T}}}{\partial t}+\boldsymbol{C}_{h h} \frac{\partial \widehat{\boldsymbol{T}}}{\partial t}\right)-\left[\left(\boldsymbol{K}_{h s} \boldsymbol{T}+\boldsymbol{K}_{h e} \widetilde{\boldsymbol{T}}+\boldsymbol{K}_{h h} \widehat{\boldsymbol{T}}\right)+\left(\boldsymbol{Q}_{h s} \boldsymbol{T}+\boldsymbol{Q}_{h e} \widetilde{\boldsymbol{T}}+\boldsymbol{Q}_{h h} \widehat{\boldsymbol{T}}\right)\right]=\boldsymbol{S}_{h}$

Here, the sub-index $s$ refers to the standard temperature degrees of freedom and sub-indexes $e$ and $h$ refer to the additional degrees of freedom associated with weak and strong discontinuities respectively. Assuming that $\mathbf{A}$ equals to $\frac{1}{\Delta t} \boldsymbol{C}-(\boldsymbol{K}+\boldsymbol{Q})$, the matrix form of the discrete problem would be: 




$\boldsymbol{T}^{n}, \widetilde{\boldsymbol{T}}^{n}$, and $\widehat{\boldsymbol{T}}^{n}$ are the values of $\boldsymbol{T}, \widetilde{\boldsymbol{T}}$ and $\widehat{\boldsymbol{T}}$ in current time step and:

$$
\begin{array}{lll}
\mathbf{A}_{s s}^{i j}=\frac{1}{\Delta t} \int_{\Omega} \rho c_{p} N_{i} N_{j} d \Omega & +\int_{\Omega} k \nabla N_{i} \cdot \nabla N_{j} d \Omega & -\int_{\Gamma_{h}} k N_{i} \cdot \nabla N_{j} \cdot n d \Gamma \\
A_{s e}^{i m}=\frac{1}{\Delta t} \int_{\Omega} \rho c_{p} N_{i} \widetilde{N}_{m} d \Omega & +\int_{\Omega} k \nabla N_{i} \cdot \nabla \widetilde{N}_{m} d \Omega & -\int_{\Gamma_{h}} k N_{i} \cdot \nabla \widetilde{N}_{m} \cdot n d \Gamma \\
A_{s h}^{i m}=\frac{1}{\Delta t} \int_{\Omega} \rho c_{p} N_{i} \widehat{N}_{m} d \Omega & +\int_{\Omega} k \nabla N_{i} \cdot \nabla \widehat{N}_{m} d \Omega & -\int_{\Gamma_{h}} k N_{i} \cdot \nabla \widehat{N}_{m} \cdot n d \Gamma
\end{array}
$$

$\mathbf{A}_{e s}^{m i}=\frac{1}{\Delta t} \int_{\Omega} \rho c_{p} \widetilde{N}_{m} N_{i} d \Omega$
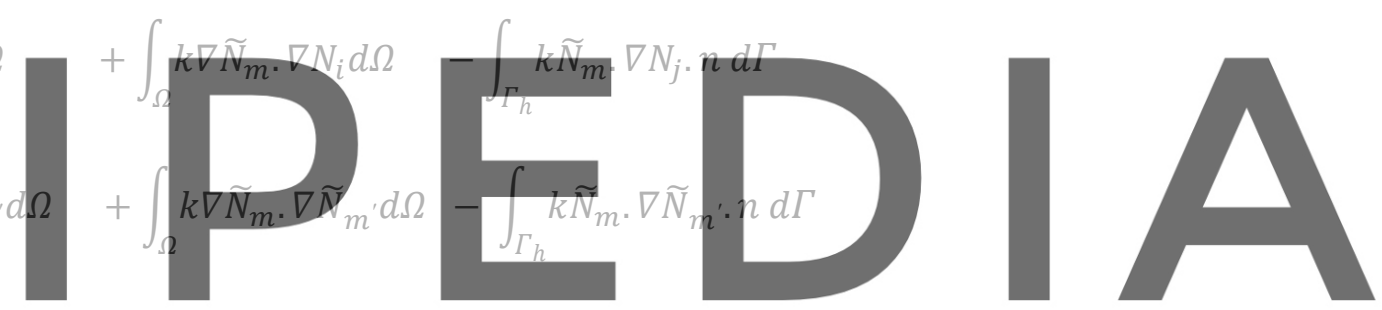

$A_{e h}^{m m^{\prime}}=\frac{1}{\int} \int \rho c_{p} \widetilde{N}_{m} \widehat{N}_{m^{\prime}} d \Omega+\int k \nabla \widetilde{N}_{m} \cdot \nabla \widehat{N}_{m^{\prime}} d \Omega-\int k \widetilde{N}_{m \cdot} \cdot \nabla \widehat{N}_{m^{\prime}} \cdot n d I$

Register for free at https//www.scipedia.com to download the version without the watermark

$$
\begin{aligned}
& \mathbf{A}_{h s}^{m i}=\frac{1}{\Delta t} \int_{\Omega} \rho c_{p} \widehat{N}_{m} N_{j} d \Omega \quad+\int_{\Omega} k \nabla \widehat{N}_{m} \cdot \nabla N_{j} d \Omega-\int_{\Gamma_{h}} k \widehat{N}_{m} \cdot \nabla N_{j} \cdot n d \Gamma \\
& \mathbf{A}_{h e}^{m m^{\prime}}=\frac{1}{\Delta t} \int_{\Omega} \rho c_{p} \widehat{N}_{m} \widetilde{N}_{m^{\prime}} d \Omega \quad+\int_{\Omega} k \nabla \widehat{N}_{m} \cdot \nabla \widetilde{N}_{m^{\prime}} d \Omega-\int_{\Gamma_{h}} k \widehat{N}_{m} \cdot \nabla \widetilde{N}_{m^{\prime}} \cdot n d \Gamma \\
& \mathbf{A}_{h h^{\prime}}^{m m^{\prime}}=\frac{1}{\Delta t} \int_{\Omega} \rho c_{p} \widehat{N}_{m} \widehat{N}_{m^{\prime}} d \Omega+\int_{\Omega} k \nabla \widehat{N}_{m} \cdot \nabla \widehat{N}_{m^{\prime}} d \Omega-\int_{\Gamma_{h}} k \widehat{N}_{m} \cdot \nabla \widehat{N}_{m^{\prime}} \cdot n d \Gamma \\
& i, j=1,3 \text { and } m, m^{\prime}=1,2
\end{aligned}
$$

Based on the fact that the enrichment functions are local to each element, we now eliminate $\widetilde{\mathbf{T}}^{\mathbf{n}}$ and $\widehat{\mathbf{T}}^{\mathrm{n}}$ at the elementary level before final assembly in the following manner:

$$
\left(\left[\begin{array}{l}
\mathbf{A}_{\text {ss }}
\end{array}\right]-\left[\begin{array}{ll}
\mathbf{A}_{\text {se }} & \mathbf{A}_{\text {sh }}
\end{array}\right]\left[\begin{array}{ll}
\mathbf{A}_{\text {ee }} & \mathbf{A}_{\text {eh }} \\
\mathbf{A}_{\text {he }} & \mathbf{A}_{\mathrm{hh}}
\end{array}\right]^{-1}\left[\begin{array}{l}
\mathbf{A}_{\text {es }} \\
\mathbf{A}_{\mathrm{hs}}
\end{array}\right]\right) \mathbf{T}^{\mathrm{n}}=\mathbf{F}+\left[\begin{array}{ll}
\mathbf{A}_{\text {se }} & \mathbf{A}_{\text {sh }}
\end{array}\right]\left[\begin{array}{ll}
\mathbf{A}_{\text {ee }} & \mathbf{A}_{\text {eh }} \\
\mathbf{A}_{\mathrm{he}} & \mathbf{A}_{\mathrm{hh}}
\end{array}\right]^{-1}\left[\begin{array}{l}
\tilde{\mathbf{F}} \\
\hat{\mathbf{F}}
\end{array}\right]
$$




\section{METHOD II}

In this case, as in the previous, new local enrichment shape functions are added. The difference is that the enrichment shape functions are defined on the basis of the standard FE shape functions as

$$
\widetilde{N}_{i}(x)=N_{i}(x) * \phi^{+(-)}
$$

where the $N_{i}(x)$ is the standard FE shape functions for node $i$ and $\phi$ is a characteristic user function as

$$
\phi^{+}=\left\{\begin{array}{ll}
0 & X \epsilon \Omega^{+} \\
1 & X \epsilon \Omega^{-}
\end{array} \quad, \quad \phi^{-}= \begin{cases}1 & X \epsilon \Omega^{+} \\
0 & X \epsilon \Omega^{-}\end{cases}\right.
$$

The advantage over alternatives is the ease of constructing such enrichment functions, which could also be readily obtained in the $3 \mathrm{D}$ case. Note that these enrichment functions are used solely for the cut elements; otherwise, only the original shape function is retrieved.

Hence, for a cut triangular element $\Omega_{\mathrm{k}}$ which is divided into two sub-elements $\Omega_{\mathrm{k}}^{+}$and $\Omega_{\mathrm{k}}^{-}$(Figure 4), the approximation form of discontinuity temperature field $T^{h}(x)$ in the finite element form can be written as

$$
\boldsymbol{T}^{h}(x)=\sum_{i=1}^{3} N_{i}(x) \boldsymbol{T}_{i}+\sum_{i=1}^{3} \widetilde{N}_{i}(x) \widetilde{\boldsymbol{T}}_{i}
$$

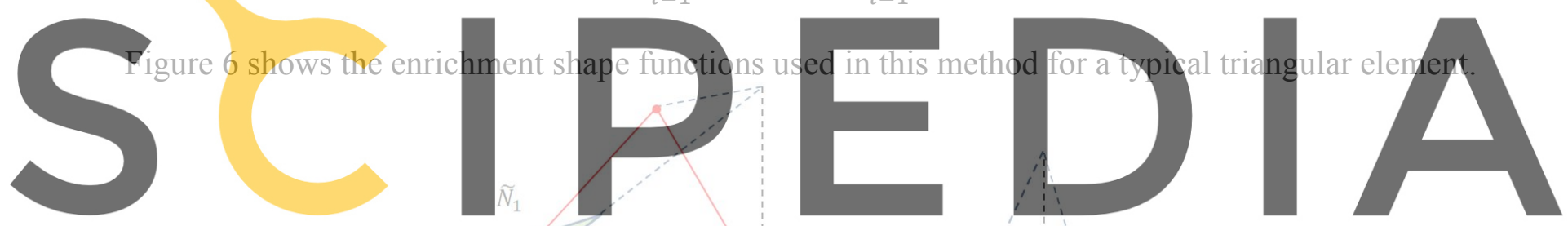

Register for free at https//www.scipedia.com to download the version without the watermark
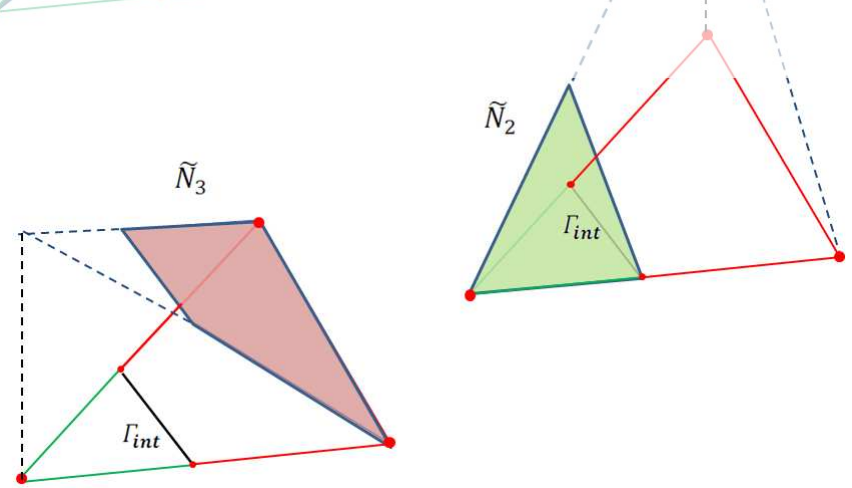

Figure 6: The enrichment function used in Method II for a triangle element

Thus, the discrete problem (5) can be decomposed using two test functions $w$ and $\widetilde{w}$, belonging to the standard and the discontinuous part respectively. The problem can be written as:

$$
\begin{gathered}
\left(\boldsymbol{C}_{e s} \frac{\partial \boldsymbol{T}}{\partial t}+\boldsymbol{C}_{e e} \frac{\partial \widetilde{\boldsymbol{T}}}{\partial t}\right)-\left[\left(\boldsymbol{K}_{e s} \boldsymbol{T}+\boldsymbol{K}_{e e} \widetilde{\boldsymbol{T}}\right)+\left(\boldsymbol{Q}_{e s} \boldsymbol{T}+\boldsymbol{Q}_{e e} \widetilde{\boldsymbol{T}}+\right)\right]=\boldsymbol{S}_{e} \\
\left(\boldsymbol{C}_{e s} \frac{\partial \boldsymbol{T}}{\partial t}+\boldsymbol{C}_{e e} \frac{\partial \widetilde{\boldsymbol{T}}}{\partial t}\right)-\left[\left(\boldsymbol{K}_{e s} \boldsymbol{T}+\boldsymbol{K}_{e e} \widetilde{\boldsymbol{T}}\right)+\left(\boldsymbol{Q}_{e s} \boldsymbol{T}+\boldsymbol{Q}_{e e} \widetilde{\boldsymbol{T}}\right)\right]=\boldsymbol{S}_{e}
\end{gathered}
$$


where the sub-indexes $s$ and $e$ refer to the standard and additional degrees of freedom respectively. The linear system can be written by blocks as:

$$
\left[\begin{array}{ll}
\mathbf{A}_{s s} & \mathbf{A}_{s e} \\
\mathbf{A}_{e s} & \mathbf{A}_{e e}
\end{array}\right] \times\left[\begin{array}{c}
\mathbf{T}^{n} \\
\widetilde{\mathbf{T}}^{n}
\end{array}\right]=\left[\begin{array}{c}
\mathbf{F} \\
\tilde{\mathbf{F}}
\end{array}\right]
$$

where $\boldsymbol{T}^{n}, \widetilde{\boldsymbol{T}}^{n}$, and $\widehat{\boldsymbol{T}}^{n}$ are the values of $\boldsymbol{T}, \widetilde{\boldsymbol{T}}$, and $\widehat{\boldsymbol{T}}$ in current time step and for $i, j=1,3$

$$
\begin{array}{lll}
\mathbf{A}_{s s}^{i j}=\frac{1}{\Delta t} \int_{\Omega} \rho c_{p} N_{i} N_{j} d \Omega & +\int_{\Omega} k \nabla N_{i} \cdot \nabla N_{j} d \Omega & -\int_{\Gamma_{h}} k N_{i} \cdot \nabla N_{j} \cdot n d \Gamma \\
\mathbf{A}_{s e}^{i j}=\frac{1}{\Delta t} \int_{\Omega} \rho c_{p} N_{i} \widetilde{N}_{j} d \Omega & +\int_{\Omega} k \nabla N_{i} \cdot \nabla \widetilde{N}_{j} d \Omega & -\int_{\Gamma_{h}} k N_{i} \cdot \nabla \widetilde{N}_{j} \cdot n d \Gamma \\
\mathbf{A}_{e s}^{i j}=\frac{1}{\Delta t} \int_{\Omega} \rho c_{p} \widetilde{N}_{i} N_{j} d \Omega & +\int_{\Omega} k \nabla \widetilde{N}_{i} \cdot \nabla N_{j} d \Omega & -\int_{\Gamma_{h}} k \widetilde{N}_{i} \cdot \nabla N_{j} \cdot n d \Gamma \\
A_{e e}^{i j}=\frac{1}{\Delta t} \int_{\Omega} \rho c_{p} \widetilde{N}_{i} \widetilde{N}_{j} d \Omega & +\int_{\Omega} k \nabla \widetilde{N}_{i} \cdot \nabla \widetilde{N}_{j} d \Omega & -\int_{\Gamma_{h}}^{k} k \widetilde{N}_{i} \cdot \nabla \widetilde{N}_{j} \cdot n d \Gamma
\end{array}
$$

As mentioned before, since the new additional functions are local to each element, which is cut, they can be statically condensed prior to assembly. Therefore, similar to the previous method, the size and the graph of the final linear system to be solved are not changed, resulting in:
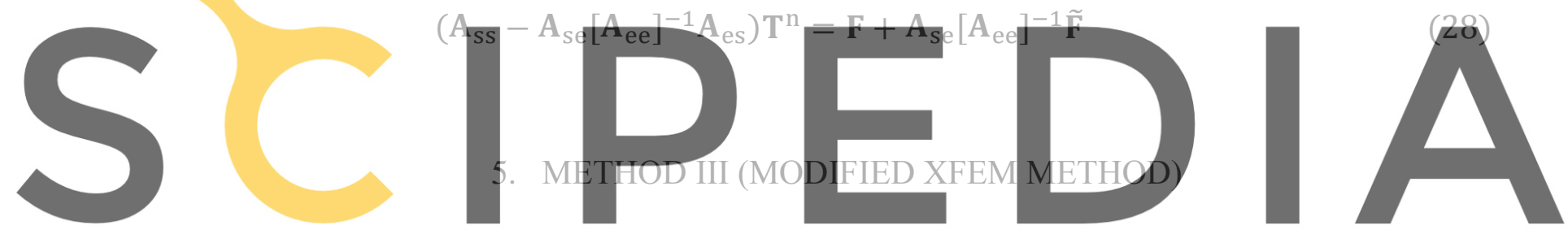

We will now try out the method proposed in [11]. Here instead of adding special functions, the FE

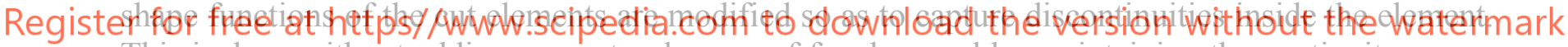

This is done without adding any extra degrees of freedom and by maintaining the continuity across

the cut borders. The modifications are local and can be computed element by element. They form a

nodal basis in the sense that they take the value one at their corresponding node and zero at the other nodes. To define them, we simply carry the value at each node towards the intersection of any edge emanating from it with the interface.

For more explanation, consider a triangular element $A B C$ that is cut by interface $\Gamma_{\text {int }}$ at two points $\mathrm{P}$ and $\mathrm{Q}$, in such a manner that it is divided into two subparts, called sub-triangle $A P Q$ and subquadrilateral $B C Q P$ (Figure 5). In this case, we assume that $\Gamma_{\text {int }}$ is a linear segment. Let $\boldsymbol{T}_{A}, \boldsymbol{T}_{B}$, and $\boldsymbol{T}_{C}$ denote the nodal values of the discrete variable $\boldsymbol{T}^{h}$, which is to be interpolated in the triangle $A B C$. Let us arbitrarily denote the triangle $A P Q$ as a 'plus' side of $\Gamma_{\text {int }}$ and quadrilateral $B C Q P$ as a 'minus' side. For the approximation to be discontinuous, on the plus side, the function $\boldsymbol{T}^{h}$ needs to be determined only by the plus node $\left(\boldsymbol{T}_{A}\right)$. Similarly, $\boldsymbol{T}^{h}$ on the minus side must depend on just minus nodes $\left(\boldsymbol{T}_{B}\right.$ and $\left.\boldsymbol{T}_{C}\right)$. The value of each point $(\mathrm{P}, \mathrm{Q})$ will be carried from the node of the original triangulation lying on the same edge and the same side. Therefore, by using this way, on the plus side of $\Gamma_{\text {int }}$, the values at $P$ and $Q$ will be $\boldsymbol{T}_{A}$, and thus $\boldsymbol{T}^{h}$ will be constant:

$$
\boldsymbol{T}_{A P Q}^{h}=\boldsymbol{T}_{A}
$$

Similarly, on the minus side, the value at $P$ will be $\boldsymbol{T}_{B}$, and the value at $Q$ will be $\boldsymbol{T}_{C}$. One can choose either to adopt a $Q_{1}$ interpolation in $B C Q P$ from these nodal values or to subdivide the 
quadrilateral into two triangles, $B C P$ and $C Q P$. For instance in the minus triangle $C Q P$, $\boldsymbol{T}^{h}$ will be the linear function that takes the value $\boldsymbol{T}_{C}$ at vertex $\mathrm{C}$, the value $\boldsymbol{T}_{C}$ at vertex $\mathrm{Q}$, and the value $\boldsymbol{T}_{B}$ at vertex P. Anyway, since the nodal values are given, the interpolation is immediate. Notice that this interpolation leads to $\boldsymbol{T}^{h}$ being discontinuous only at $\Gamma_{\boldsymbol{i n t}}$. This is because the function $\boldsymbol{T}^{h}$, which is restricted to any edge of the triangle, is uniquely determined by the values at the nodes lying at the endpoints of that edge.

The basic functions $N_{A}, N_{B}$, and $N_{C}$ are defined to be piecewise affine inside each of the subtriangles, namely $A P Q, B C P$, and $C Q P$. It only remains to define their values at the vertices of the sub-triangles, i.e. at the points $A, B, C, P$, and $Q$. However, as they are discontinuous at $\Gamma_{\text {int }}$, two values (plus and minus) are given at the points $P$ and $Q$. These values are

$\begin{array}{lll}N_{A}(A)=1 & N_{B}(A)=0 & N_{C}(A)=0 \\ N_{A}(B)=0 & N_{B}(B)=1 & N_{C}(B)=0 \\ N_{A}(C)=0 & N_{B}(C)=0 & N_{C}(C)=1 \\ N_{A}\left(P^{+}\right)=1 & N_{B}\left(P^{+}\right)=0 & N_{C}\left(P^{+}\right)=0 \\ N_{A}\left(P^{-}\right)=0 & N_{B}\left(P^{-}\right)=1 & N_{C}\left(P^{-}\right)=0 \\ N_{A}\left(Q^{+}\right)=1 & N_{B}\left(Q^{+}\right)=0 & N_{C}\left(Q^{+}\right)=0 \\ N_{A}\left(Q^{-}\right)=0 & N_{B}\left(Q^{-}\right)=0 & N_{C}\left(Q^{-}\right)=1\end{array}$

The significant feature of this method is that unlike two previous methods, the shape functions are $C^{0}$ across the cut borders (but not across $\Gamma_{\text {int }}$ )

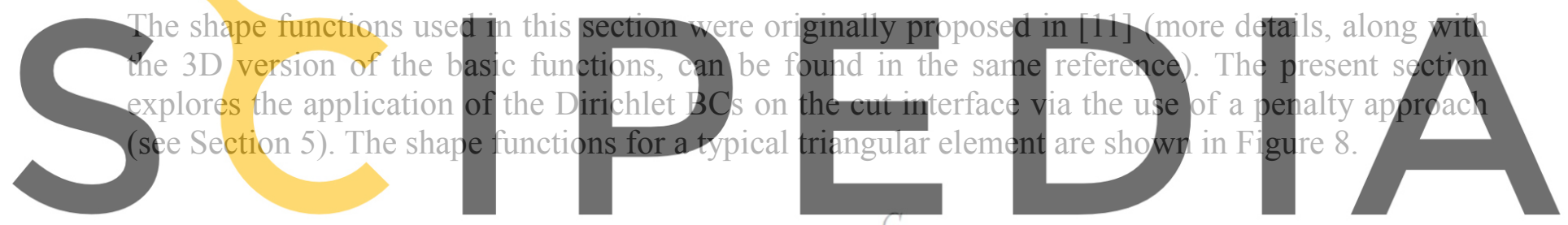

Register for free at https//www.scipedia.com to download the version without the watermark



Figure 7: Partition of a single finite element into sub-elements following the interface PQ

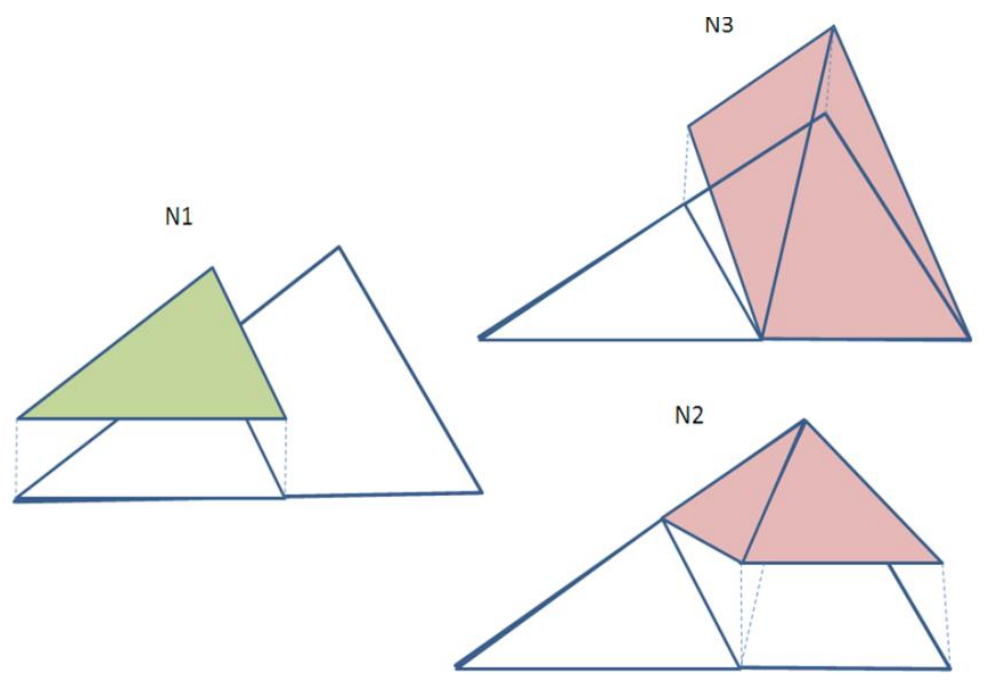

Figure 8: Modified shape functions for the discontinuous space without additional unknowns (used in Method III) 


\section{IMPOSING BOUNDARY CONDITIONS ON DISCONTINUOUS INSIDE THE ELEMENT(INTERFACE)}

Imposing the Neumann-type boundary condition on the interface that cuts the elements is straightforward, since it is needed to compute the integral of the flux over the interface. In particular, for the application of an adiabatic boundary condition (zero heat flux), the terms including the flux at the interface, $\boldsymbol{S}$, can even be omitted.

The imposition of the Dirichlet boundary conditions on $\Gamma_{i n t}$ is, on the other hand, a little more involved since no node is present on the boundary $\Gamma_{\text {int }}$ to strongly apply the condition. Many specific techniques for the implementation of the Dirichlet boundary conditions in X-FEM have been proposed, such as the Lagrange multiplier method [12-15], the penalty method [16], and Nitsche's method [17-19]. In our case, the Lagrange multiplier method is considered for the first two methods, while the penalty method is chosen for the third method in order to ensure that our enriched variables assume the desired value on the boundary.

\section{1: Implementation of Lagrange Multiplier in Method I}

In Method I, four additional degrees of freedom as Lagrangian multipliers are used in order to impose the Dirichlet constraint, e.g. a zero value of the temperature at the interface. Let us consider a triangular FE mesh containing discontinuity $\Gamma_{\text {int }}$. As we know, the function is discontinuous at the interface that we have decomposed into two sub-interfaces $\Gamma_{\text {int }}{ }^{+}$and $\Gamma_{\text {int }}{ }^{-}$(see Figure 9). This approach is implemented by using two integral points on each sub-interface $\Gamma_{\text {int }}{ }^{+}$and $\Gamma_{\text {int }}{ }^{-}$.

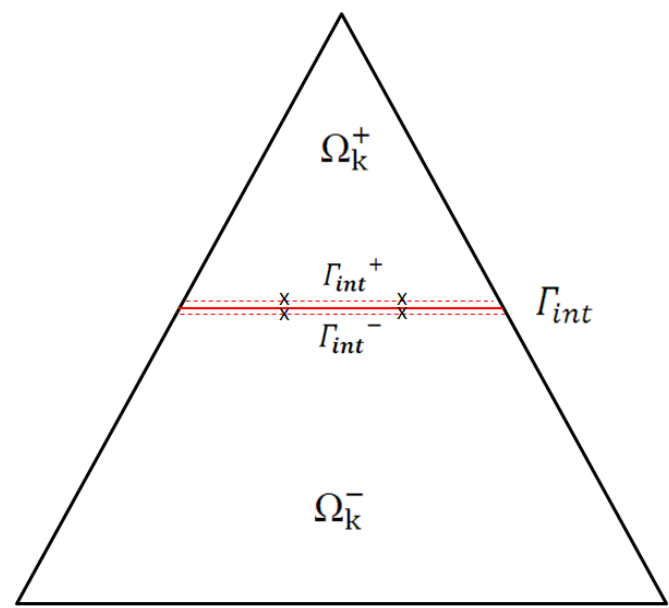

Figure 9: A triangle element containing $\Gamma_{\text {int }}$ with two gauss points on each sub-element $\Gamma_{\text {int }}{ }^{+}$and $\Gamma_{\text {int }}{ }^{-}$

Hence, after adding the Lagrange multiplier term to the equation (21), the system formed by the enrichment variables and the Lagrange multipliers can be written by blocks in the following manner: 


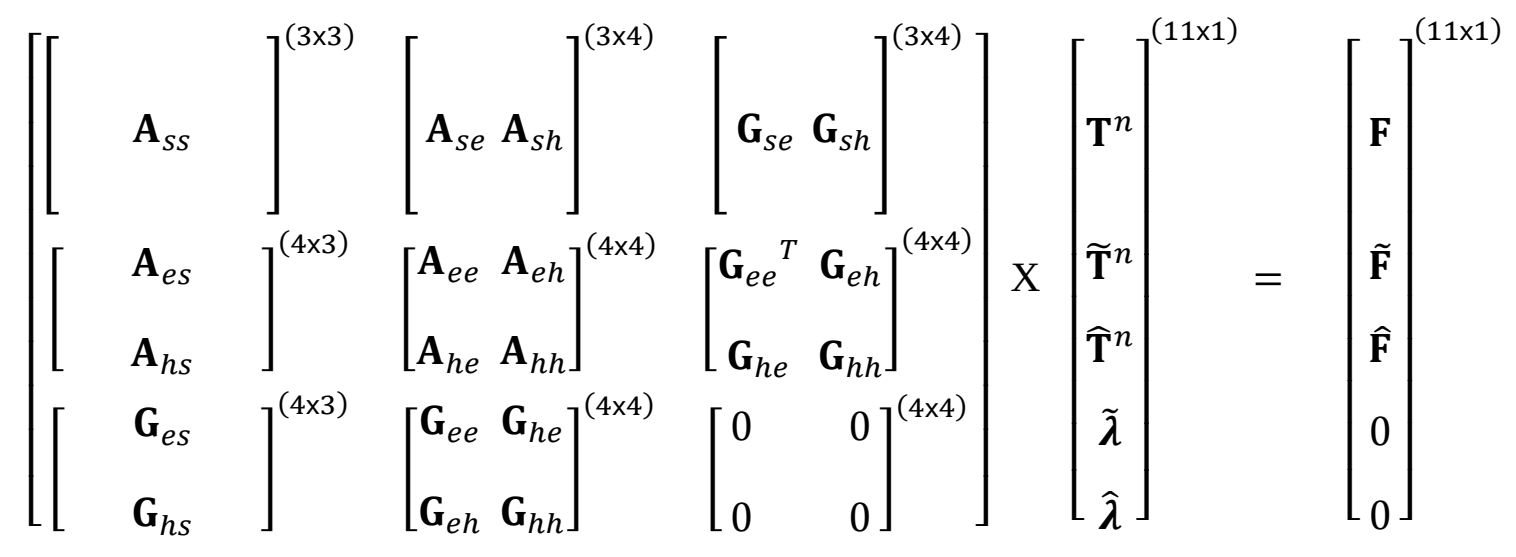

where

$$
\begin{aligned}
& G_{h s}^{i j}:=\int_{\Gamma_{i n t}{ }^{+}} \widehat{N}^{i} N^{j} \partial \Gamma_{i n t}{ }^{+}+\int_{\Gamma_{i n t^{-}}} \widehat{N}^{i} N^{j} \partial \Gamma_{i n t}{ }^{-} \quad i=1,2 @ j=1,3 \\
& G_{e s}^{i j}:=\int_{\Gamma_{i n t^{+}}} \widetilde{N}^{i} N^{j} \partial \Gamma_{i n t}{ }^{+}+\int_{\Gamma_{i n t^{-}}} \widetilde{N}^{i} N^{j} \partial \Gamma_{i n t}{ }^{-} \quad i=1,2 @ j=1,3 \\
& G_{e h}^{i j}:=\int_{\Gamma_{i n t}{ }^{+}}^{i n t} \widetilde{N}^{i} \widehat{N}^{j} \partial \Gamma_{i n t}{ }^{+}+\int_{\Gamma_{i n t}{ }^{-}} \widetilde{N}^{i} \widehat{N}^{j} \partial \Gamma_{i n t}{ }^{-} \quad i, j=1,2 \\
& G_{h h}^{i j}:=\int_{\Gamma_{i n t}{ }^{+}}^{n n t} \widehat{N}^{i} \widehat{N}^{j} \partial \Gamma_{i n t}{ }^{+}+\int_{\Gamma_{i n t}{ }^{-}} \widehat{N}^{i} \widehat{N}^{j} \partial \Gamma_{i n t}{ }^{-} \quad i, j=1,2 \\
& G_{e e}^{i j}:=\int_{\Gamma_{i n t}{ }^{+}} \widetilde{N}^{i} \widetilde{N}^{j} \partial \Gamma_{i n t}^{+}+\int_{\Gamma_{i n t}{ }^{-}} \widetilde{N}^{i} \widetilde{N}^{j} \partial \Gamma_{i n t}{ }^{-} \quad i, j=1,2
\end{aligned}
$$

The important point is that in this case, as our enrichment is local, we can also define the additional Lagrangian multipliers locally to the enriched element, and statically condense the system formed by the enrichment variables and the Lagrange multipliers. Note that this is not generally possible (since the diagonal block corresponding to the Lagrange multipliers is zero). It is possible in our case solely because we are condensing out at once both our local enrichment unknowns and the Lagrangian multiplier. The obvious advantage is that the global system matrix is not modified, while the local contributions are adjusted to enforce the desired Dirichlet constraint. The result after condensation is as follows:

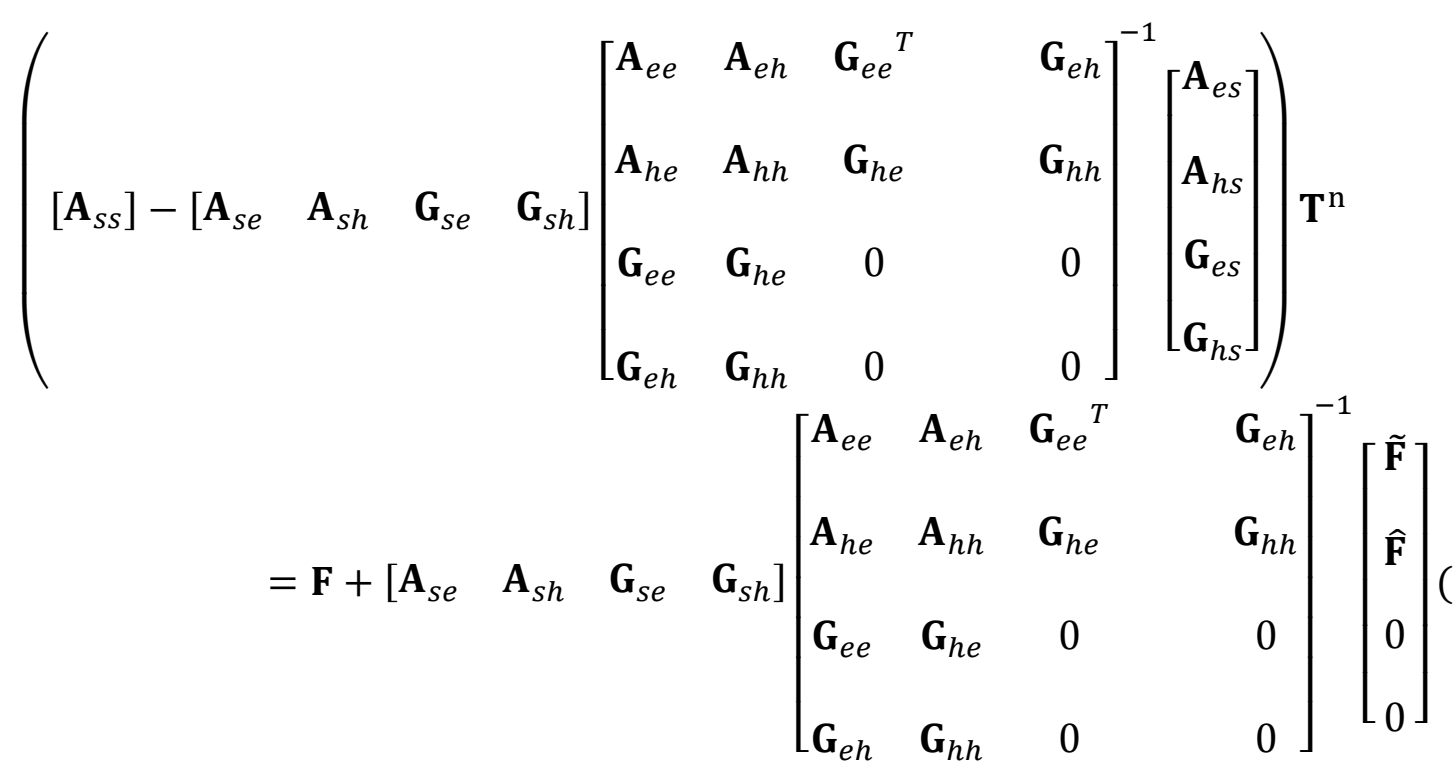




\section{2: Implementation of Lagrange Multiplier in Method II}

In this method, due to the fact that three enrichment functions are added, three degrees of freedom are used as Lagrangian multipliers, in order to impose the Dirichlet constraint.

Thus, the matrix formed by the system (27) may be rewritten as

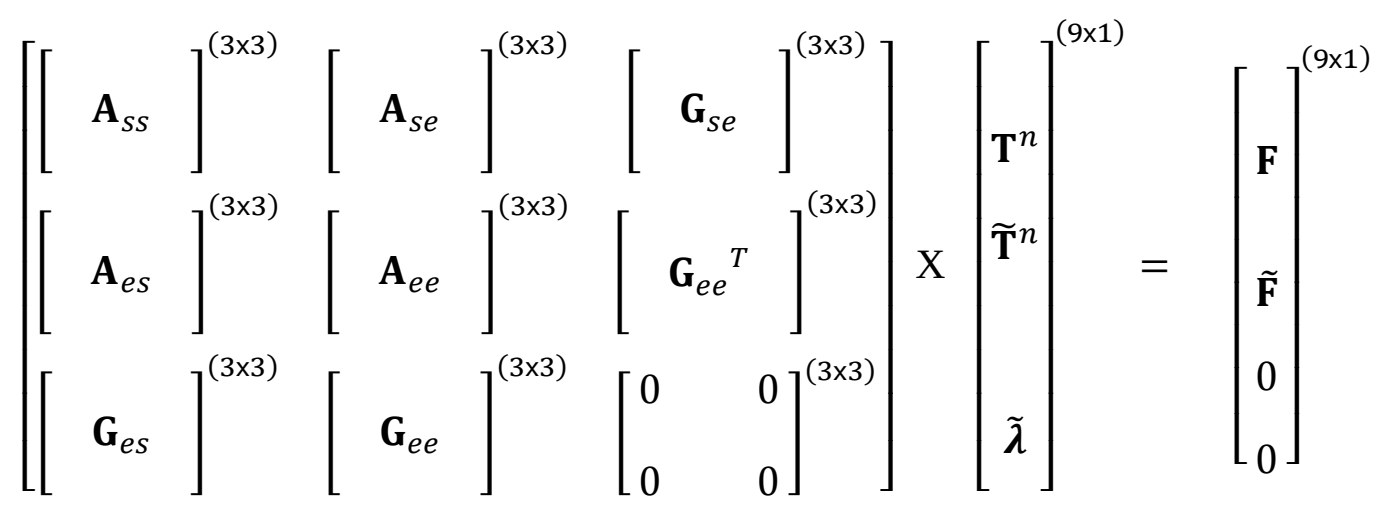

where

$$
\begin{aligned}
& G_{e s}^{i j}:=\int_{\Gamma_{i n t}{ }^{+}} \widetilde{N}^{i} N^{j} \partial \Gamma_{i n t}{ }^{+}+\int_{\Gamma_{\text {int }}{ }^{-}} \widetilde{N}^{i} N^{j} \partial \Gamma_{i n t}{ }^{-} \quad i, j=1,3 \\
& G_{s e}^{i j}=G_{e s}^{j i} i, j=1,3 \\
& G_{e e}^{i j}:=\int_{\Gamma_{\text {int }}{ }^{+}} \widetilde{N}^{i} \widetilde{N}^{j} \partial \Gamma_{i n t}{ }^{+}+\int_{\Gamma_{\text {int }}{ }^{-}} \widetilde{N}^{i} \widetilde{N}^{j} \partial \Gamma_{i n t}{ }^{-} \quad i, j=1,3
\end{aligned}
$$

\section{3: Implementation of Penalty in Method III}

As stated earlier, in Method III no enrichment functions are added to capture discontinuities (see Section 5). The practical consequence is that the static condensation procedure employed for the first two alternatives is not viable here. For this reason, a penalty method is chosen to enforce the Dirichlet boundary condition. For more explication, if the liner equation is $\mathbf{K T}=\mathbf{F}$, then the penalty method can apply the boundary condition using a large number $\alpha$ to modify the stiffness matrix as well as the right-hand side vector as

$$
(\mathbf{K}+\alpha \widetilde{K}) \mathbf{T}=\mathbf{F}+\alpha \widetilde{F}
$$

where

$$
\begin{aligned}
& \widetilde{K}=\int_{\Gamma_{\text {int }}{ }^{+}} \widetilde{N}^{T} \widetilde{N} \partial \Gamma_{\text {int }}{ }^{+}+\int_{\Gamma_{\text {int }}{ }^{-}} \widetilde{N}^{T} \widetilde{N} \partial \Gamma_{\text {int }}{ }^{-} \\
& \tilde{F}=\int_{\Gamma_{\text {int }}{ }^{+}} \widetilde{N}^{T} T_{\text {int }} \partial \Gamma_{\text {int }}{ }^{+}+\int_{\Gamma_{\text {int }}{ }^{-}} \widetilde{N}^{T} T_{\text {int }} \partial \Gamma_{\text {int }}{ }^{-}
\end{aligned}
$$




\section{EXAMPLES SECTION}

\section{Example 1}

Here a heat conduction domain $\Omega:=(0,6) \times(0,6)$ cut by an immersed discontinuity $\Gamma_{\text {int }}$ is considered (see Figure 10). If the thermal conductivity $\mathrm{K}$ for the entire domain was one and the temperature was set at ten at the top boundary and twenty at the bottom, then the analytical exact solution where a zero-prescribed temperature is imposed at the interface $\left(T_{\text {int }}=0\right)$ would be:

$$
\left\{\begin{array}{cl}
T_{1}-\left(\frac{T_{1}}{e}\right) \times x & \text { for } x<e \\
0 & \text { for } x=e \\
\frac{T_{2}}{(X-e)} \times(x-e) & \text { for } x>e
\end{array}\right.
$$

Figures 11 and 12 show the behaviour of the temperature obtained from Methods I, II, and III in comparison with the exact solution on a coarse mesh. Note that the temperature distribution exactly matches the expected one outside the cut elements. The behaviour of the contour lines within the cut elements is a pure visualization artefact. In this area, the post-processor assumes that the results will always be interpolated linearly from the nodal results, thus producing the shown distribution that is unable to take into account the presence of the enrichment functions.



Figure 10: The geometric and boundary conditions for a heat conduction domain with immersed discontinuity (Mesh size $=1.0)$ 


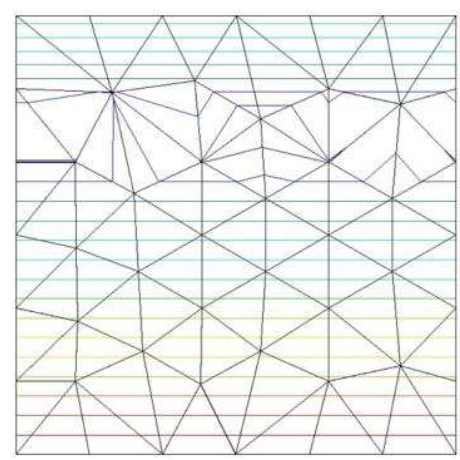

Exact Solution

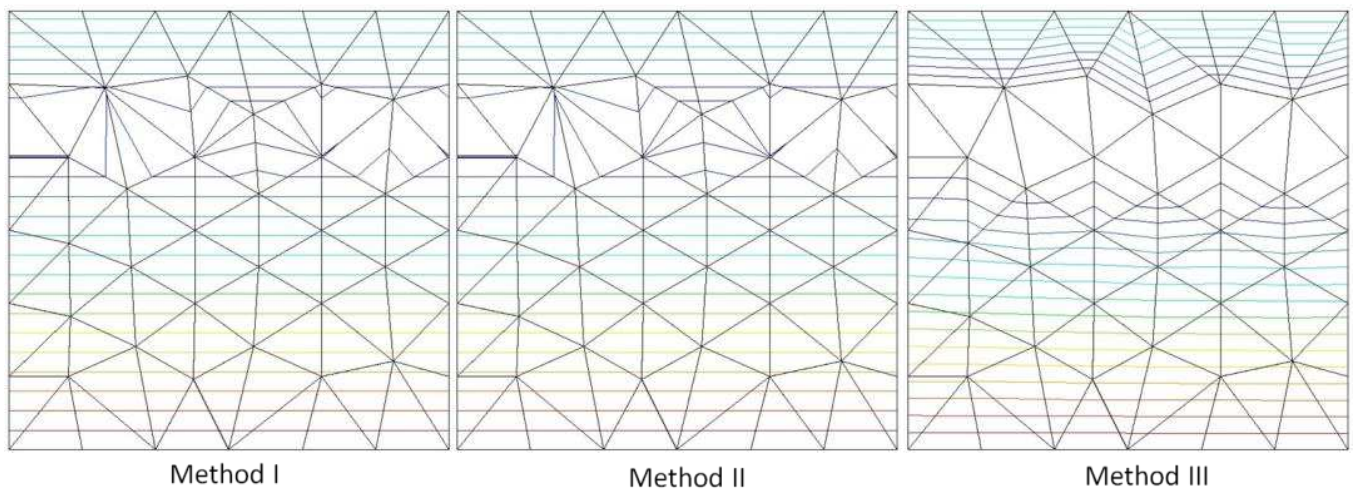

Figure 11: Contour lines of the temperature where a zero-prescribed temperature is imposed at the interface

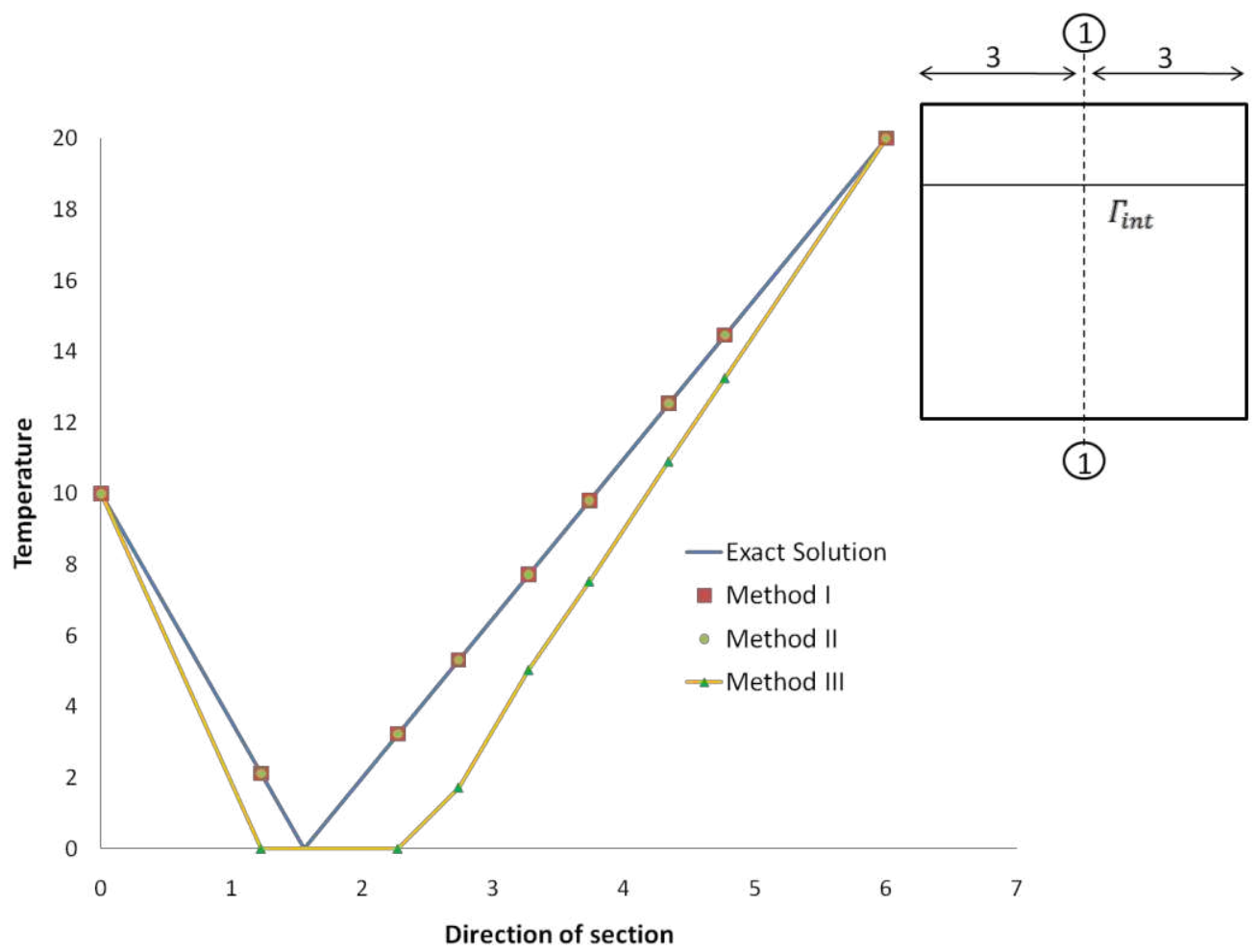

Figure 12: Comparison of the temperature fields of Methods I, II, and III with the exact solution where the value of the temperature (say zero) is imposed at the interface $($ Mesh size $=1.0)$ 


\section{$\mathrm{L}_{2}$ Errorvs $\mathrm{h}$}

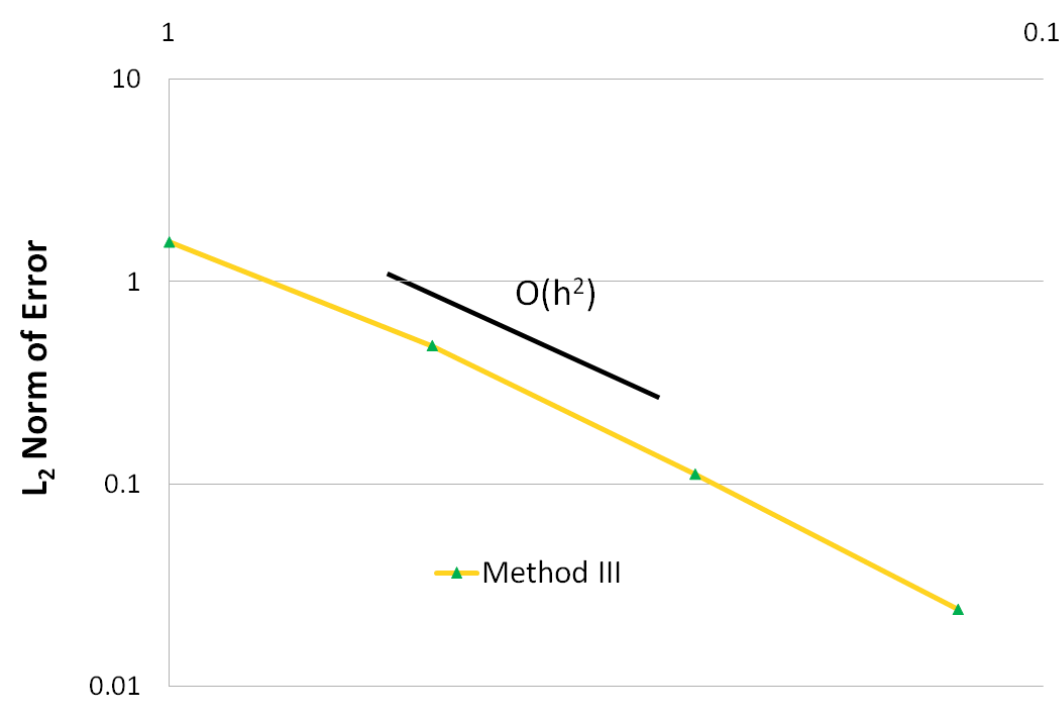

Figure 13: Convergence rate of error in L2-norm for the nodal temperature of the elements with respect to the mesh iterative in Method III

As we see in Figure 11, the result obtained from Methods I and II are exact, while Method III shows an acceptable result (the interpolation error in the $\mathrm{L}_{2}(\Omega)$-norm is of order $h^{2}$, Figure 13). This means if the temperature is set at zero at the interface, in Method III the value of the temperature at each original node of the element has to be zero due to its shape function. In contrast, in Methods I and II, the values of the temperature at each original node are obtained so that the temperature is zero at the interface.

\section{Example 2}

We now consider a stationary heat conduction domain similar to the previous example with the same boundary conditions, but the discontinuity is immersed within the domain (see Figure 14). Both temperature (Dirichlet boundary condition) and its gradient (Neumann boundary condition) are enforced to be zero separately at the $\Gamma_{\text {int }}$. The results obtained from the proposed methods are compared with the results of the classic FE method where the discontinuity is matched by the mesh (see Figure 15). These comparisons are shown in Figures 16-23. 


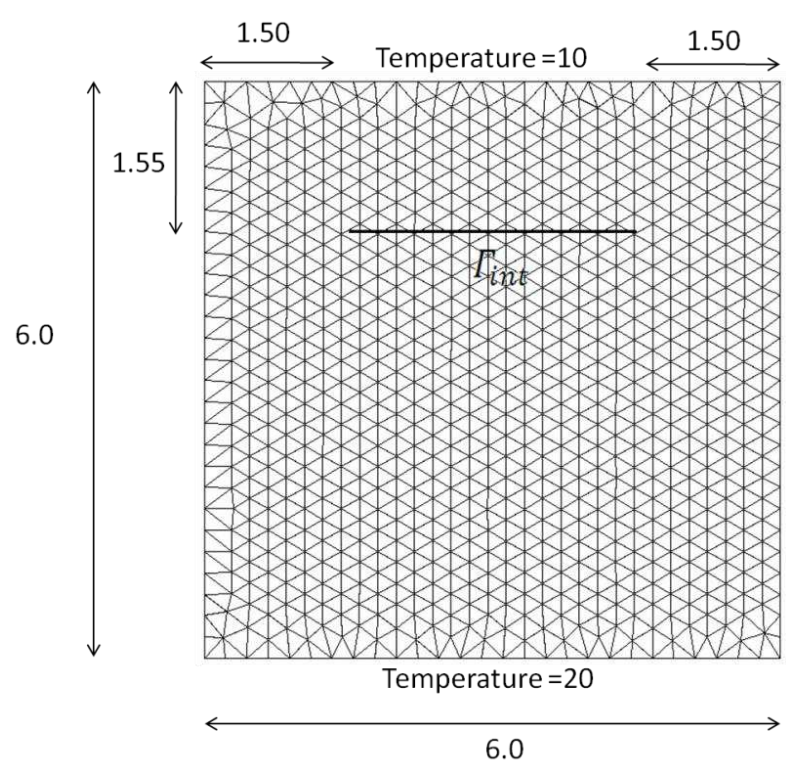

Figure 14: Mesh and boundary conditions for the thermal domain with an embedded interface that does not conform to the element edges $($ Mesh size $=0.22)$

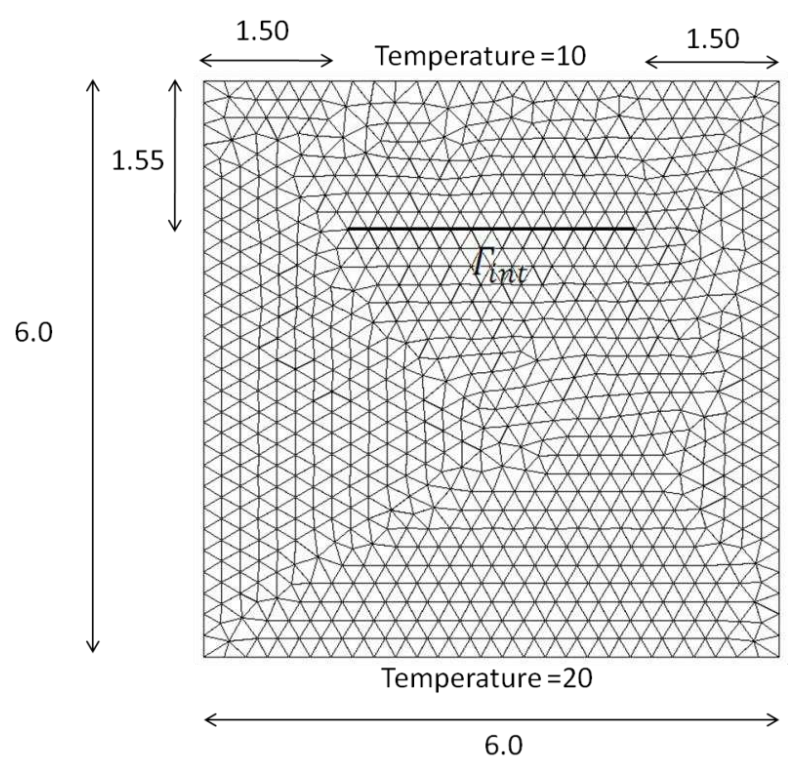

Figure 15: Mesh and boundary conditions for the thermal domain with an interface (gap) fitted to the mesh (Mesh size = 0.22 ) 


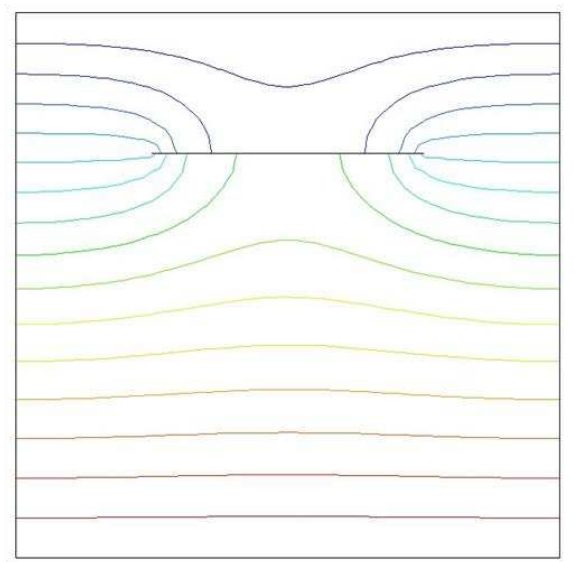

Finite Element Method

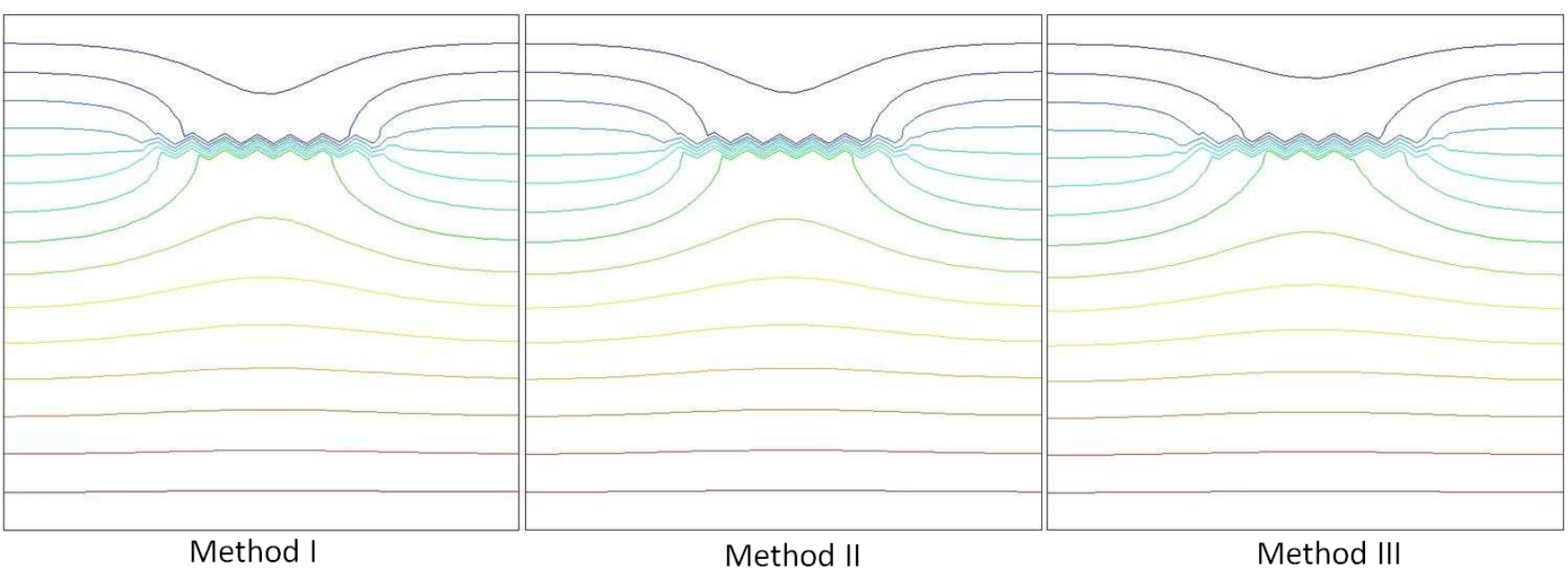

Figure 16: Contour lines of temperature where the Neumann boundary condition is imposed $(\nabla T \cdot n=0)$ at the interface (Mesh size equals to 0.22 )

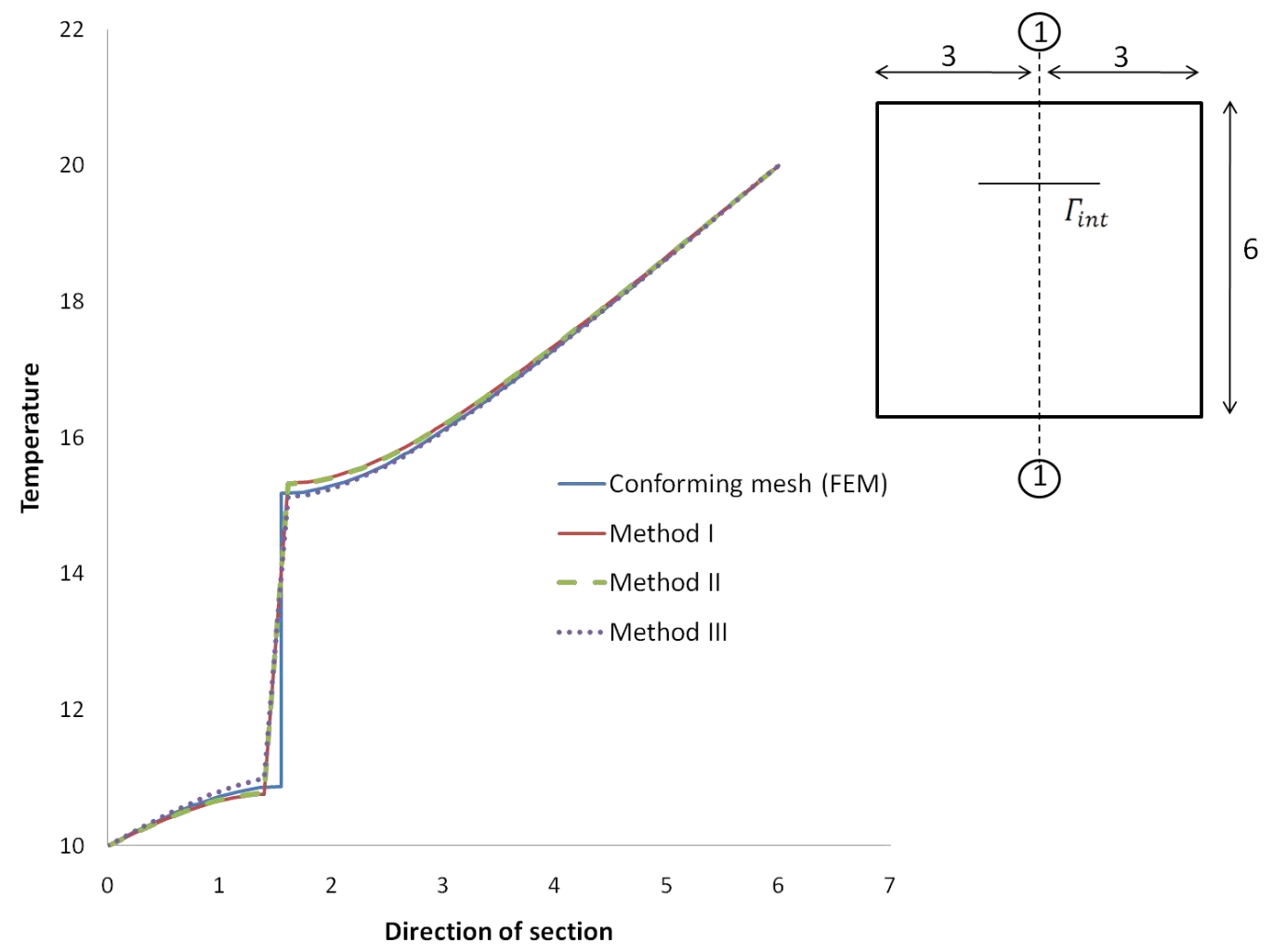

Figure 17: Comparison of the temperature field at section 1-1 between Methods I, II, III, and the classic FE method $($ Mesh size $=0.22)$ 


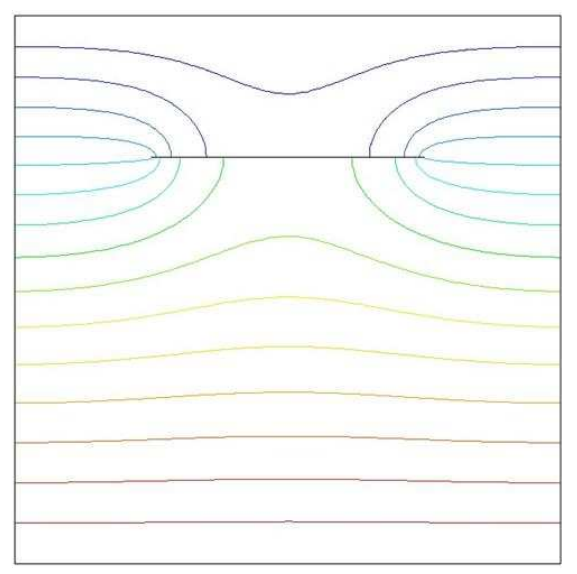

Finite Element Method

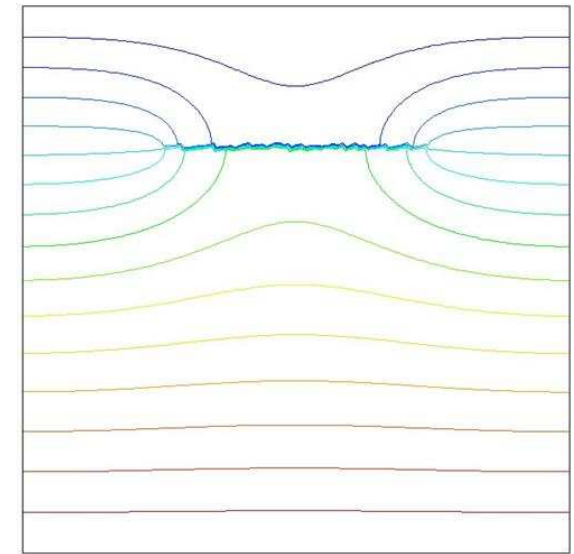

Method I

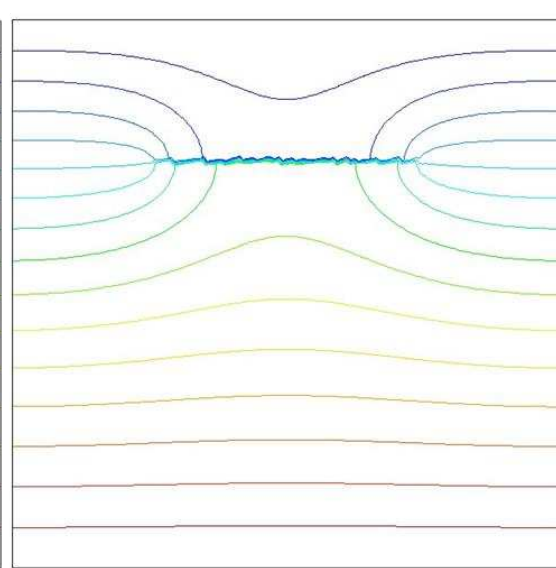

Method II

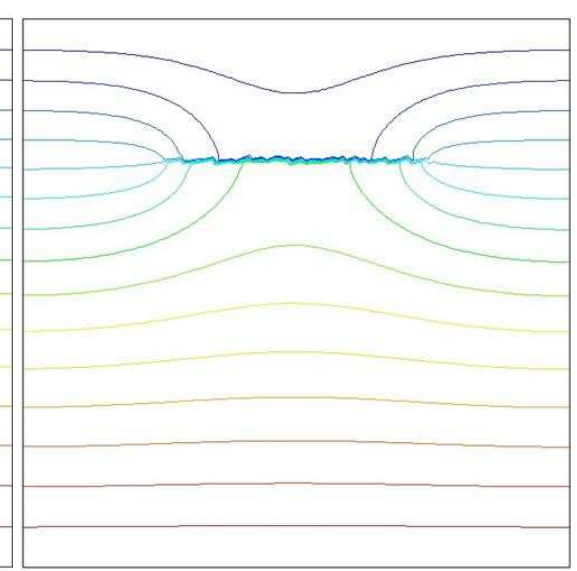

Method III

Figure 18: Contour lines of temperature where the Neumann boundary condition is imposed $(\nabla T . n=0)$ at the interface (Mesh size equals to 0.055 )



Figure 19: Comparison of the temperature field at section 1-1 between Methods I, II, III, and the classic FE method $($ Mesh size $=0.055)$ 


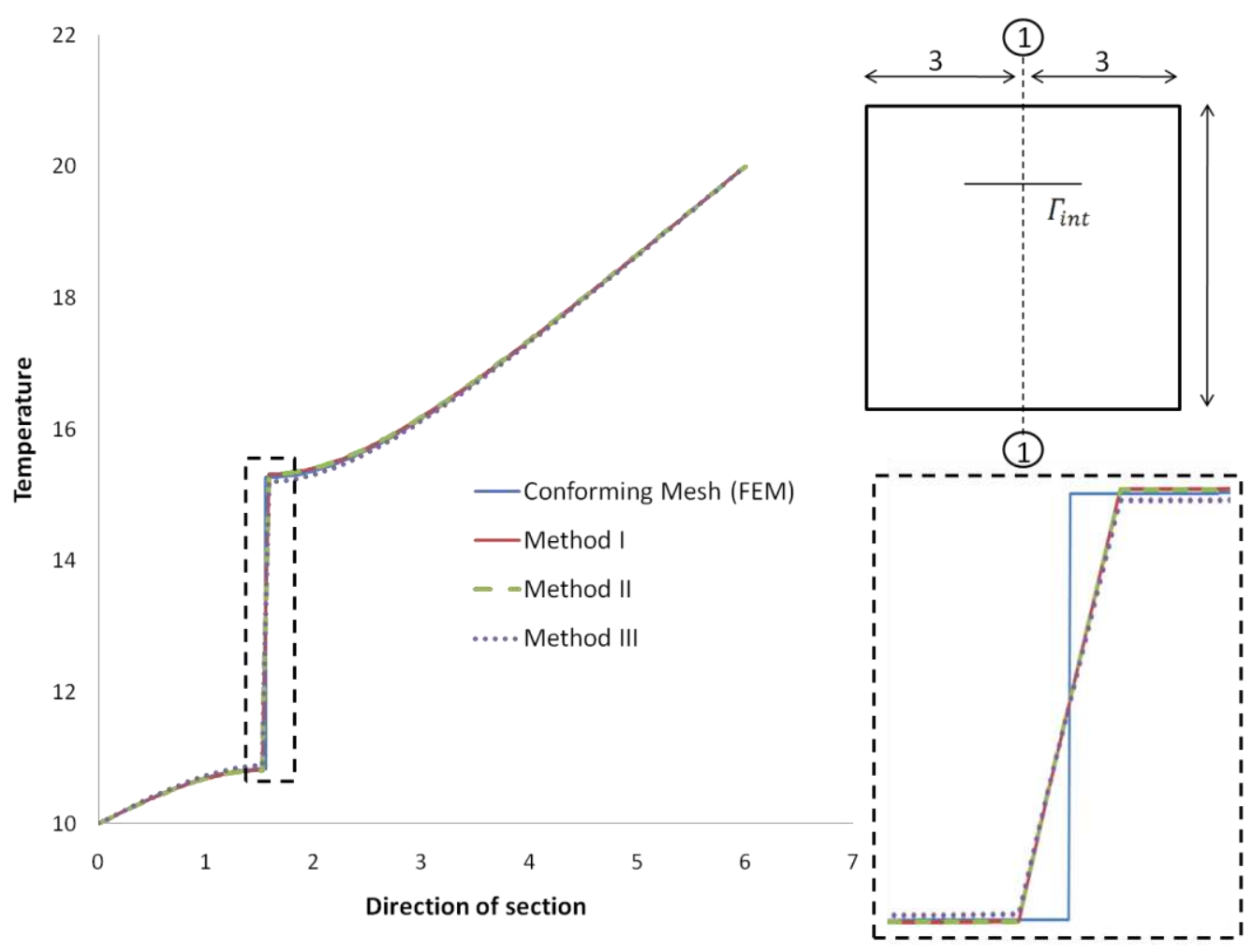

Figure 20: Contour lines of temperature where the value of the temperature is imposed as zero $(T=0)$ at the interface as Dirichlet boundary condition (Mesh size $=0.22$ )

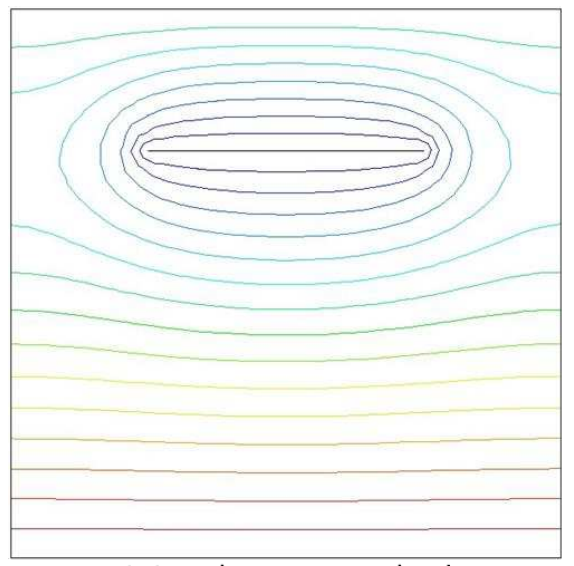

Finite Element Method



Method I

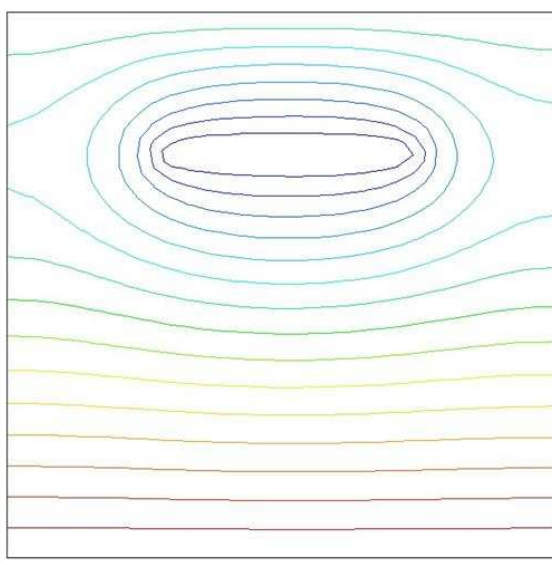

Method II

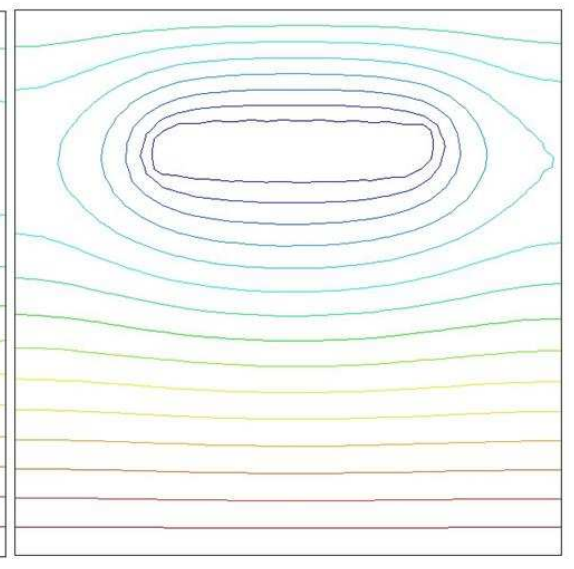

Method III

Figure 21: Temperature field (at section 1-1) where the value of temperature is imposed as zero at the interface (Mesh 


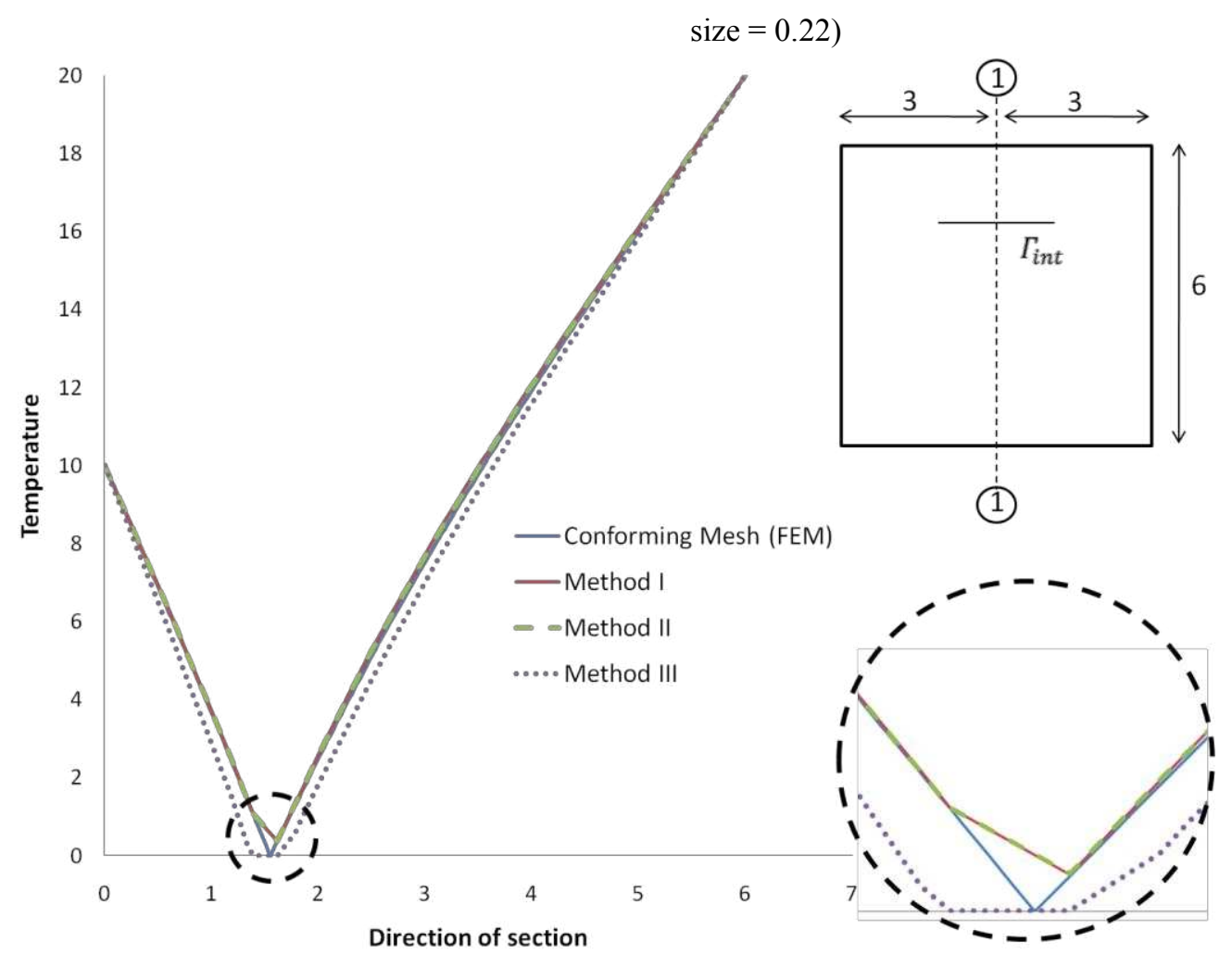

Figure 22: Contour lines of temperature where the temperature value is imposed as zero $(T=0)$ at the interface as Dirichlet boundary condition (Mesh size $=0.055$ )

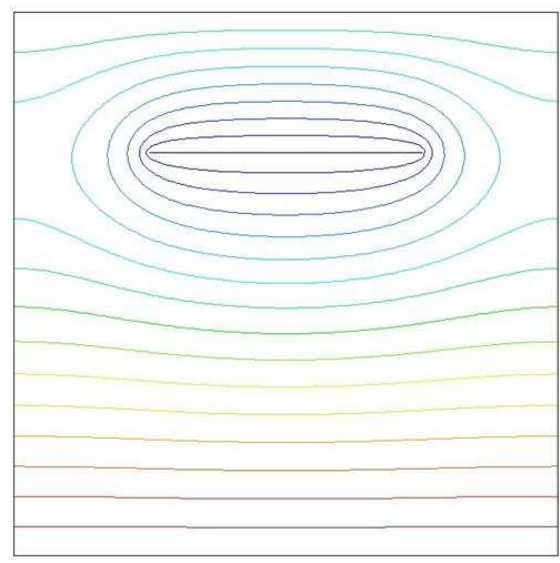

Finite Element Method

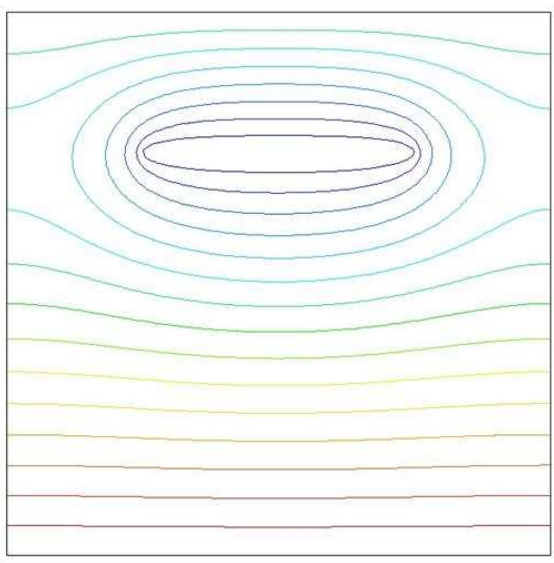

Method I

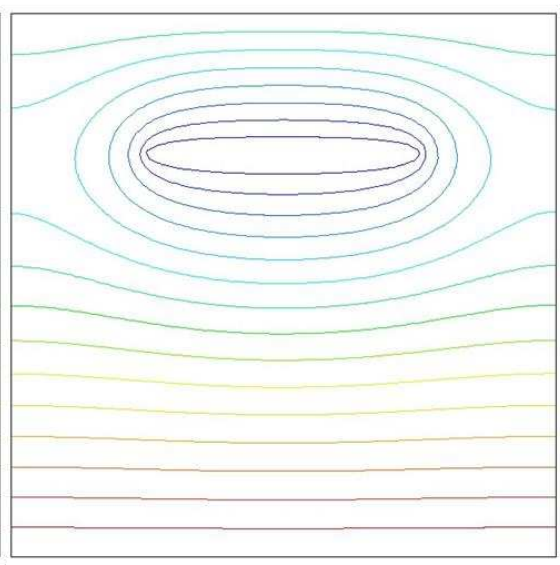

Method II

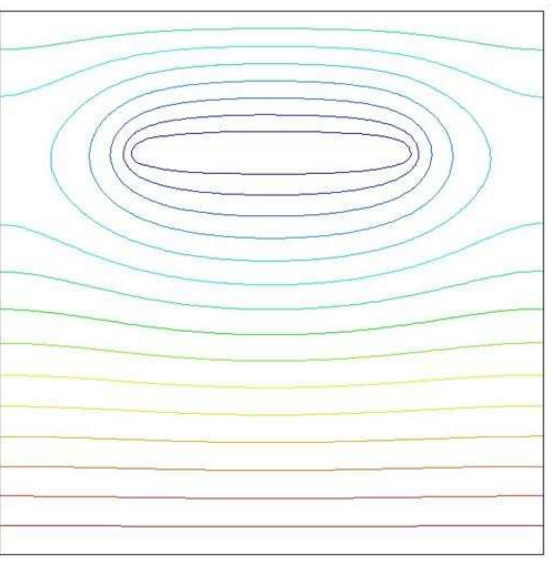

Method III

Figure 23: The temperature field (at section 1-1) where the temperature value is imposed as zero at the interface (Mesh size $=0.055)$ 
As we can see from the results, when discontinuity immerses with an adiabatic boundary condition for temperature, Method III has better convergence than the others in a coarse mesh, while Methods I and II have shown better results when the size of the mesh decreases. However, when a zeroprescribed temperature (Dirichlet boundary condition) is imposed at the interface, the first two proposed methods are shown more accurately than the third one in either coarse or fine mesh. This result agrees with our expectation. For more explanation, this poor convergence in Method III happens due to the concept of modified shape function, which is used in this method (see Section 5). Therefore, the value of temperature is zero not only at the interface but also on the whole cut element. In contrast, in Methods I and II the temperature of the triangle nodes is calculated so that the value of the temperature can be zero exactly at the interface.

\section{Example 3}

As a third experience, we investigate the heat conduction problem in a domain with the same geometry, but an embedded diagonal discontinuity cuts the domain. The mesh and the boundary conditions for the domain are shown in Figure 24. The gradient of the temperature is set at zero on the embedded discontinuity. At the end, the results from the proposed methods are compared with the reference solution where the discontinuity is fitted to the mesh (see Figures 25-31).

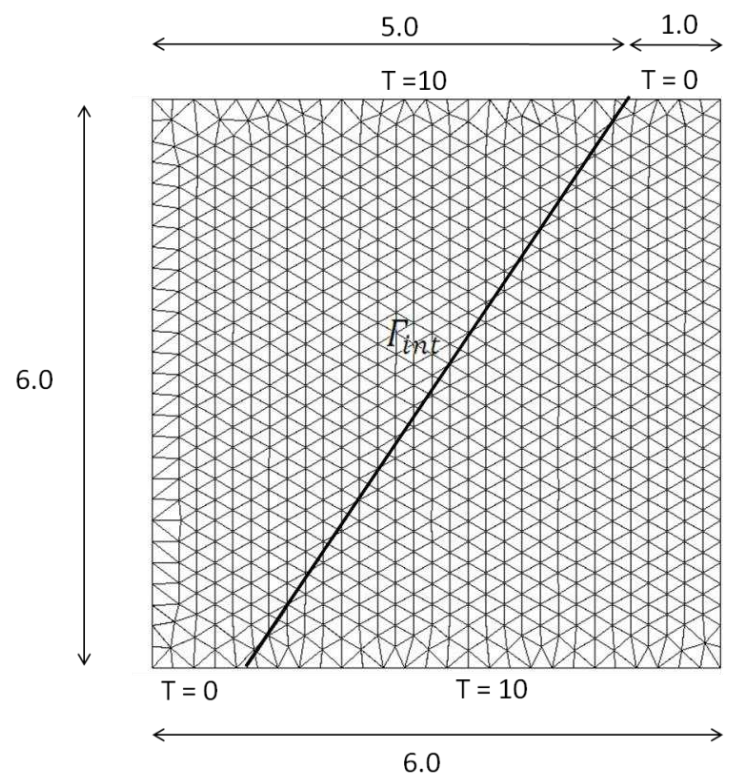

Figure 24: The mesh and the boundary conditions for the domain with an embedded diagonal discontinuity through it $($ Mesh size $=0.22)$ 


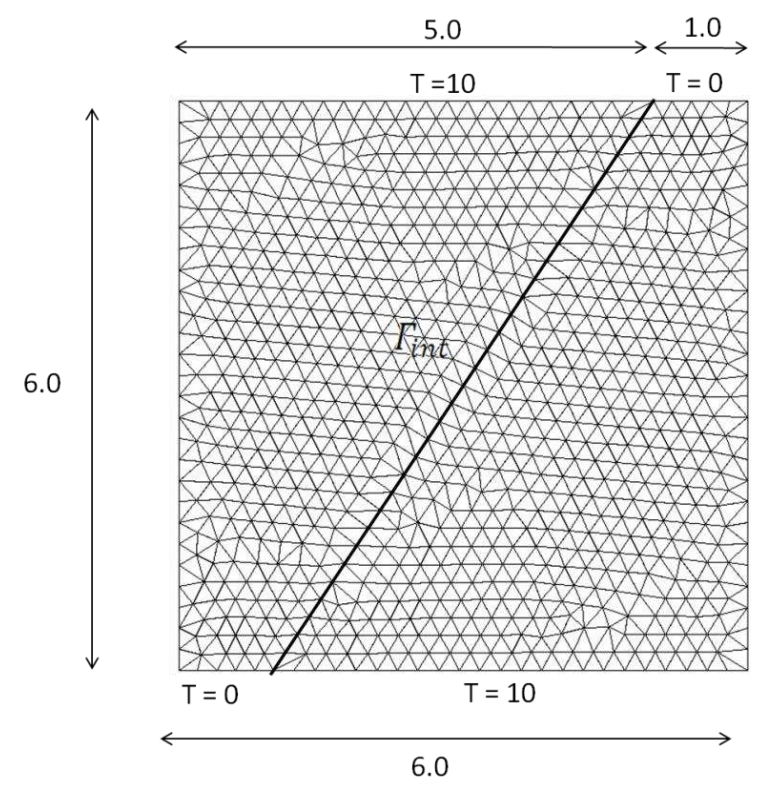

Figure 25: The mesh and the boundary conditions for the domain with a gap (discontinuity) that is fitted to the mesh $($ Mesh size $=0.22)$

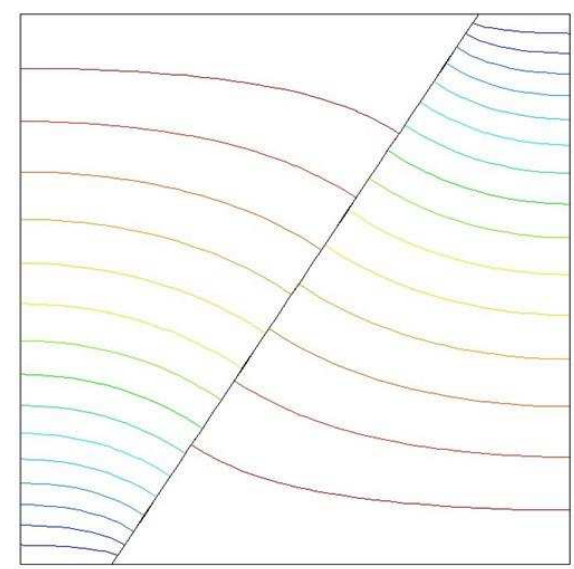

Finite Element Method

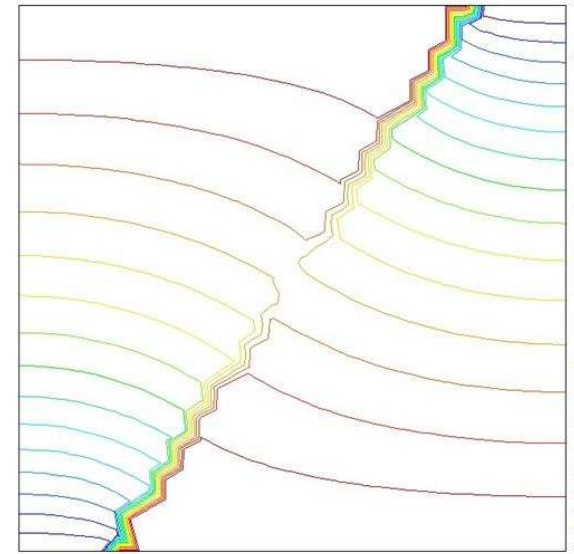

Method I



Method II



Method III

Figure 26: Contour lines of the temperature field when discontinuity is immersed with an adiabatic boundary condition for temperature (Mesh size equals to 0.22 ) 




Finite Element Method

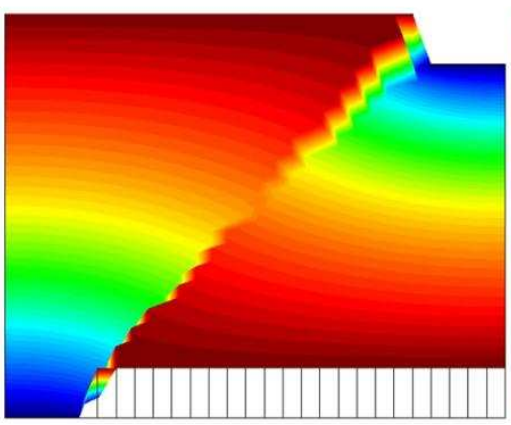

Method I



Method II

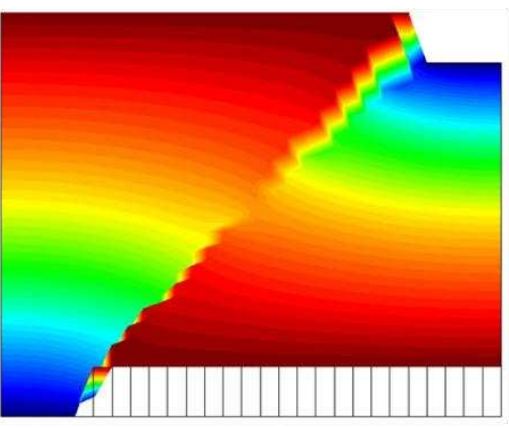

Method III

Figure 27: The discontinuities of the temperature field where the Neumann boundary condition is imposed

(Mesh size equals to 0.22 )

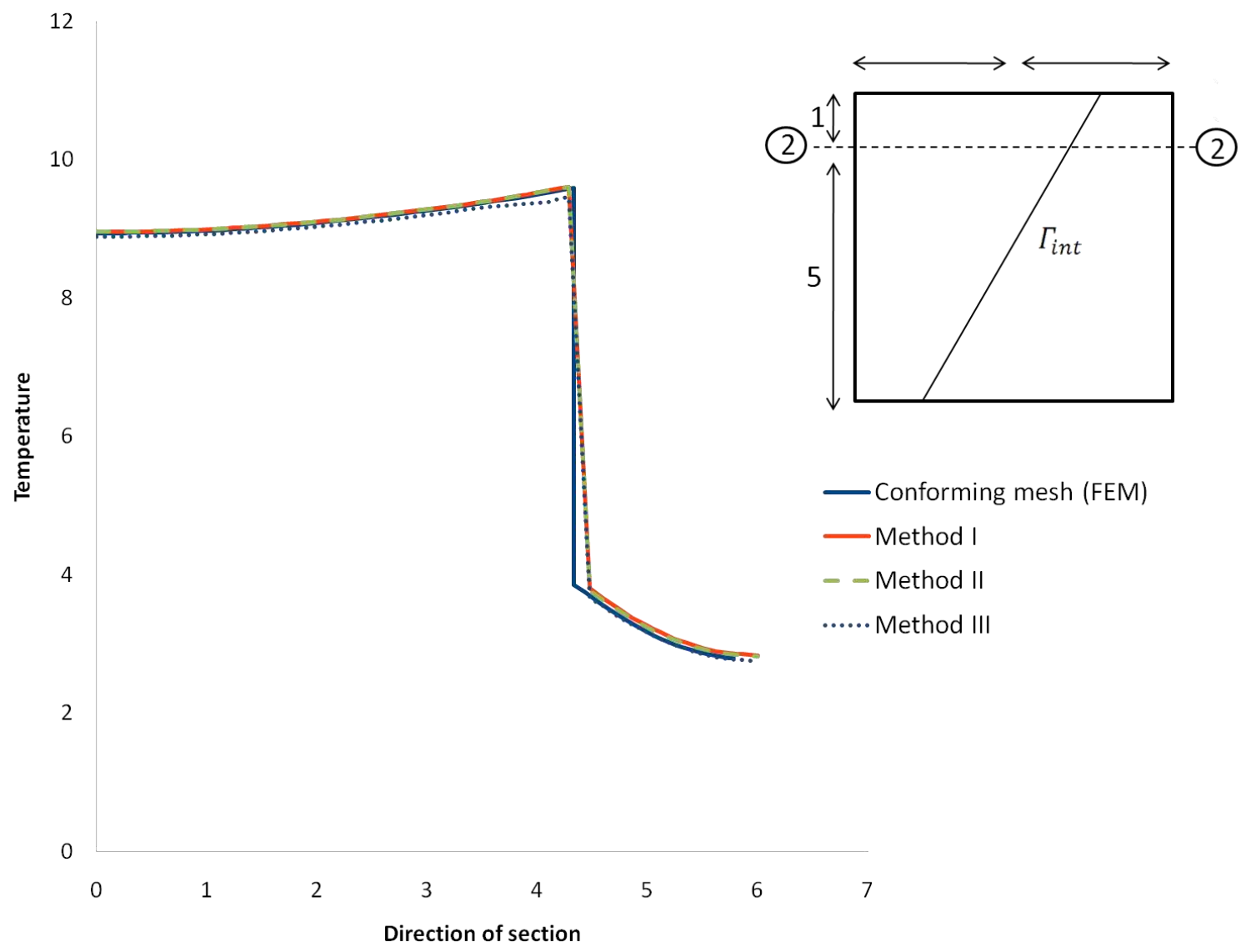

Figure 28: Comparison of the temperature field at section 2-2 between the proposed methods and the classic FE method $($ Mesh size $=0.22)$ 


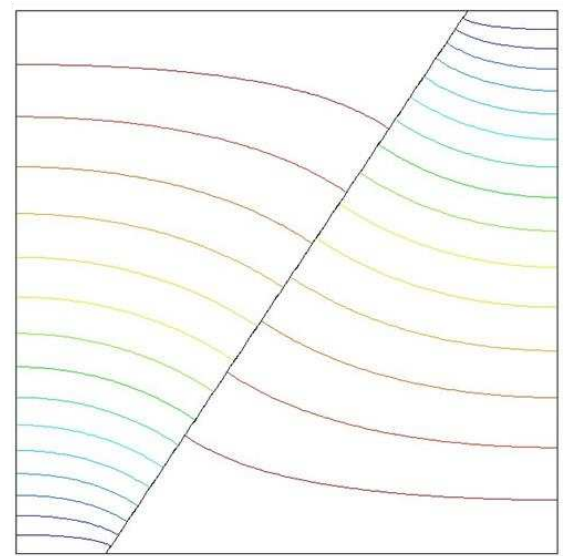

Finite Element Method

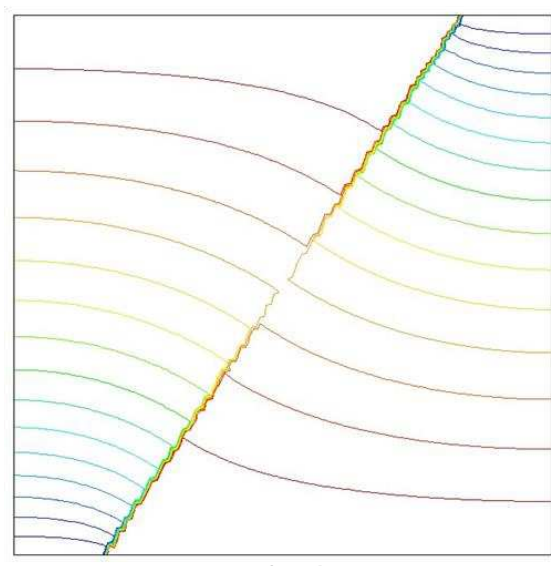

Method I

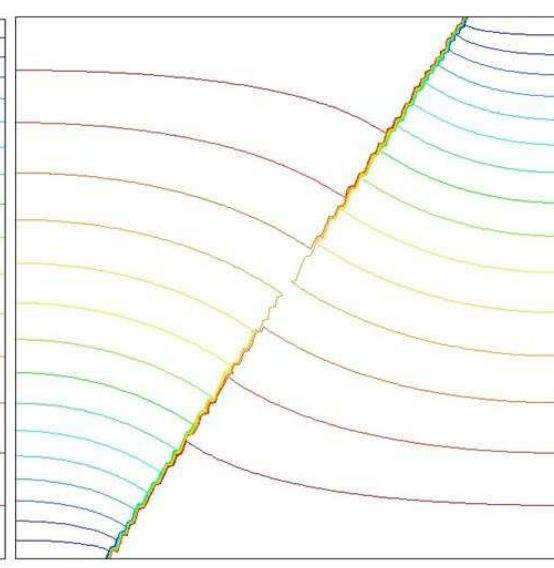

Method II

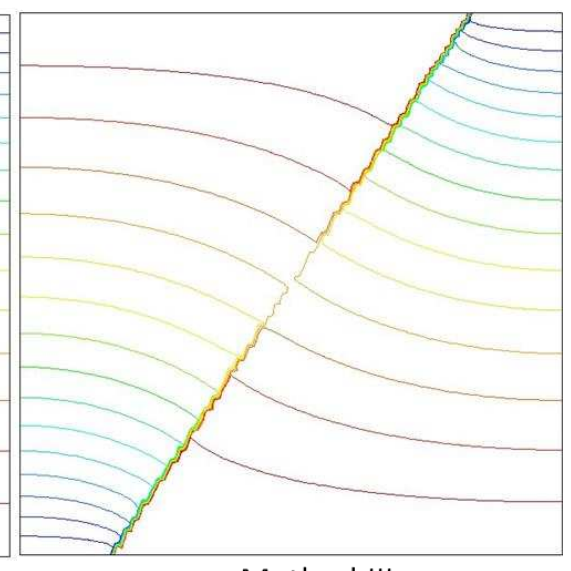

Method III

Figure 29: Contour lines of the temperature field when the discontinuity is immersed with an adiabatic boundary condition for temperature (Mesh size equals to 0.055)

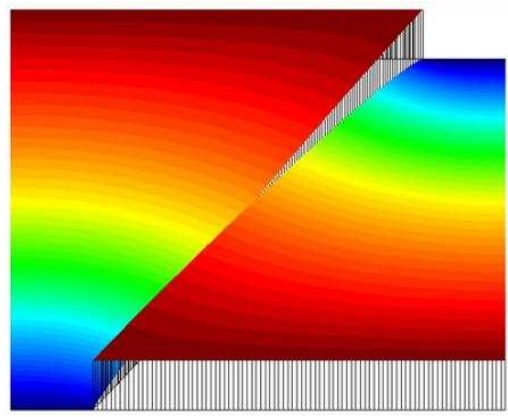

Finite Element Method



Method I

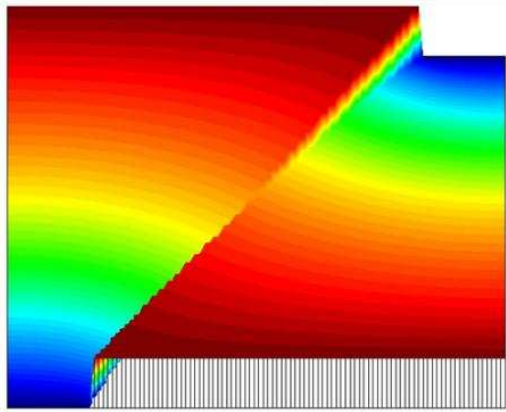

Method II



Method III

Figure 30: The discontinuities of the temperature field where the Neumann boundary condition is imposed at the interface (The mesh size equals to 0.055 ) 


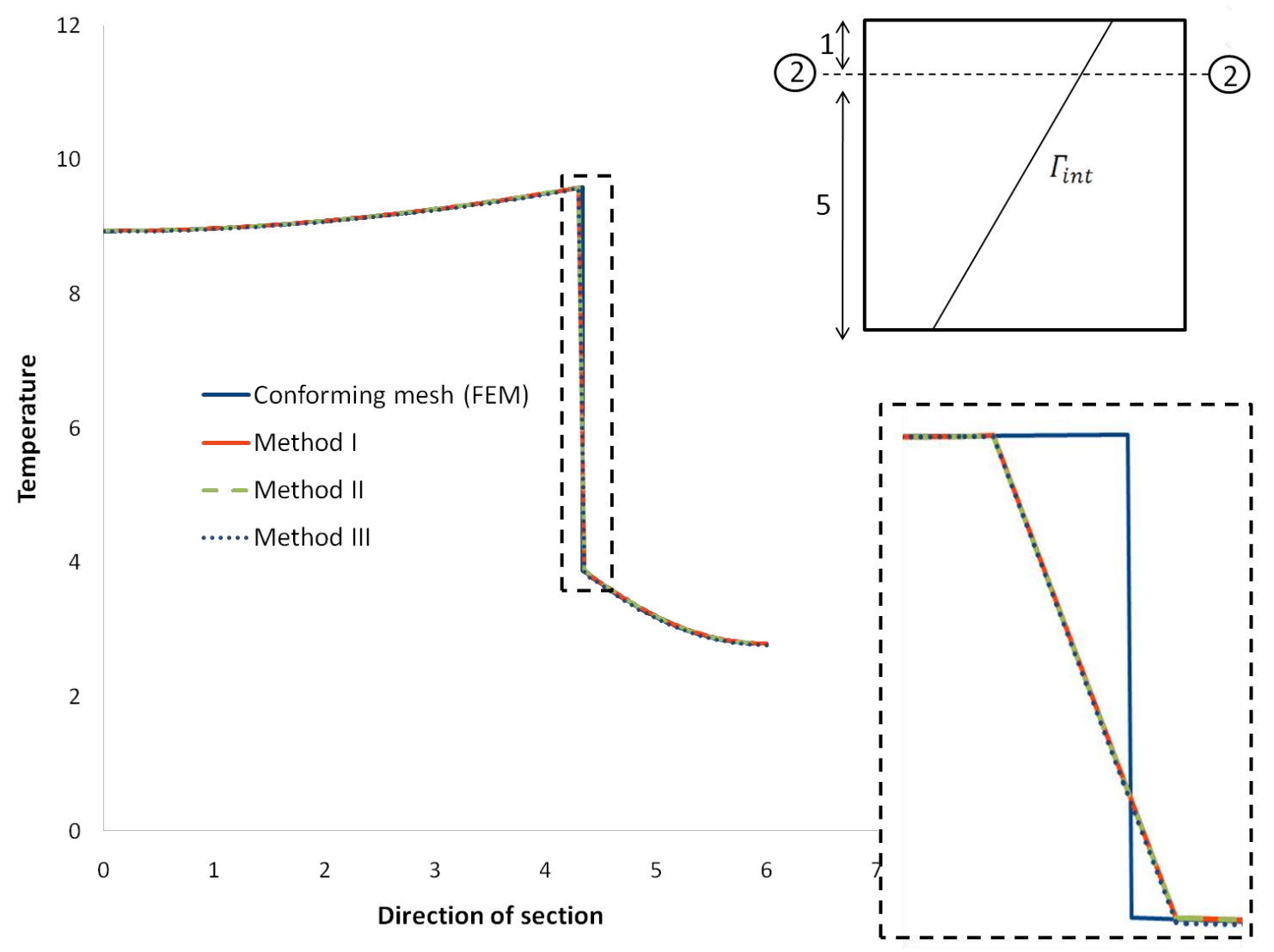

Figure 31: Comparison of the temperature field at section 2-2 between the proposed methods and the classic FE method (The mesh size $=0.055)$

\section{$\mathrm{L}_{2}$ Error vs $\mathrm{h}$}

1



Figure 32: Convergence rate of the error in L2-norm for the nodal temperature of the elements with respect to the mesh iterative in Methods I, II, and III 
As is evident from Figure 32, when results obtained from the domain with a gap (discontinuity is fitted to the mesh) are selected as reference solutions, the convergence rate of the interpolation error for Methods I and II shows higher order than Method III.

\section{Example 4}

In this example, we consider a heat conduction flow in which the computational domain contains a curved discontinuity instead of a straight one. Similar to previous examples, a value of one is chosen for thermal conductivity $\mathrm{K}$ for the entire the domain. The temperature at the top and the bottom boundary are set at 10 and 20 respectively (see Figure 33). Then both the Neumann and the Dirichlet boundary conditions are imposed at the embedded interface separately. The temperature field obtained from our proposed methods is compared with that corresponding to the classic FE method where the mesh is body-fitted to the gap geometry (see Figure 34). The results are shown in Figures 35-42. It is clear that, although all methods have shown an acceptable behaviour near the interface (except at the tips of the embedded interface), Methods I and II exhibit significantly better behaviour than Method III. Note that at the tips of the interface, the proposed methods exhibit difference from the conforming mesh method. This difference occurs due to the failure to choose the cut elements at the tips. In other words, only the elements that are cut completely are enriched, while those containing the tips may not be cut by the interface completely. Hence, these elements are considered normal, non-cut elements.

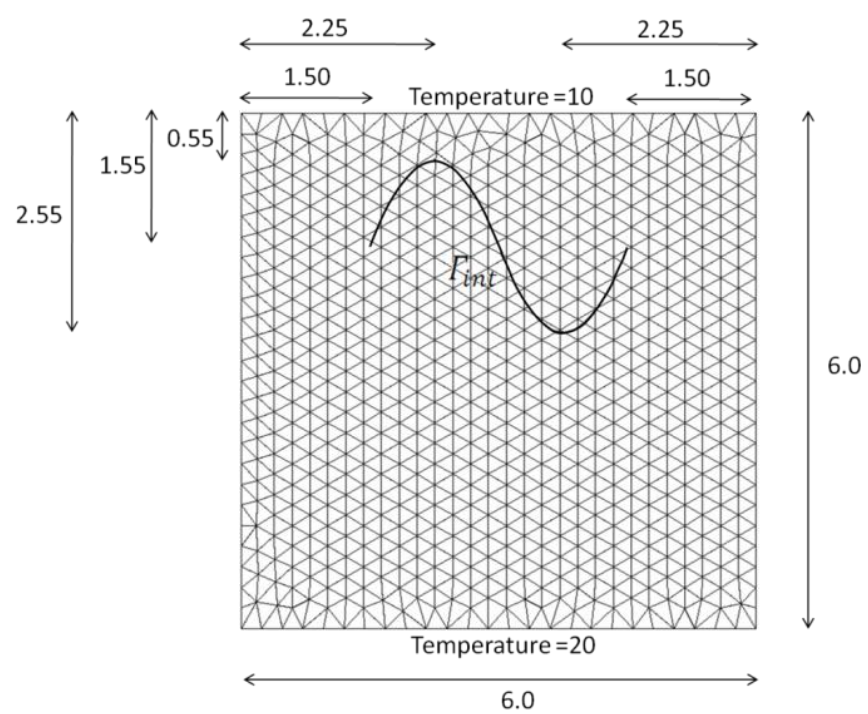

Figure 33: The mesh and the boundary conditions for the flow domain with a curved discontinuity not fitted to the mesh $($ Mesh size $=0.22)$ 


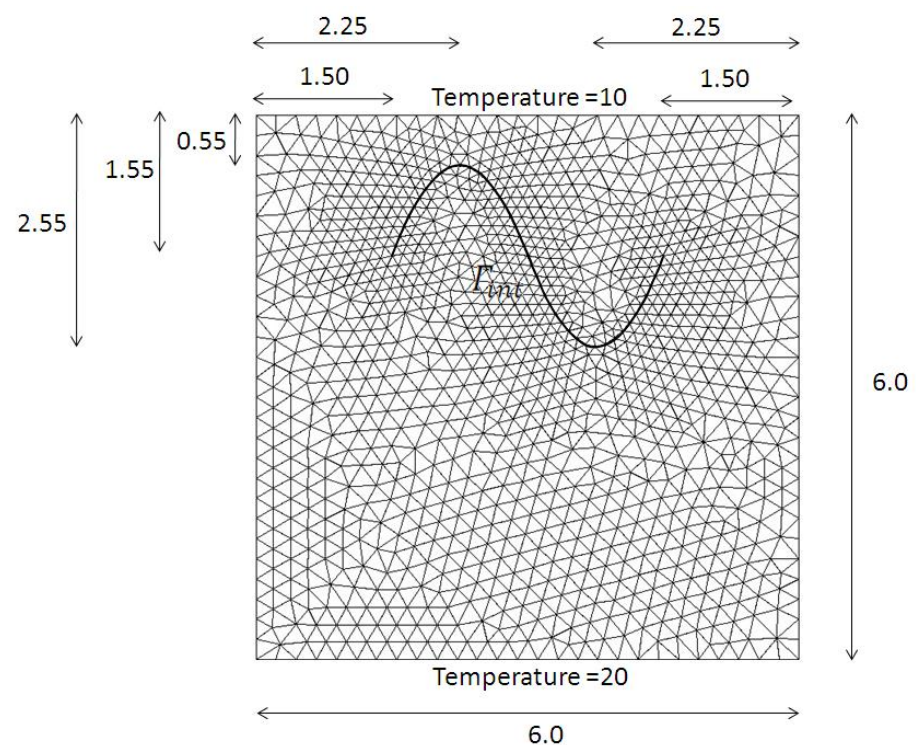

Figure 34: The mesh and the boundary conditions for the flow domain with a gap fitted to the mesh $($ Mesh size $=0.22)$

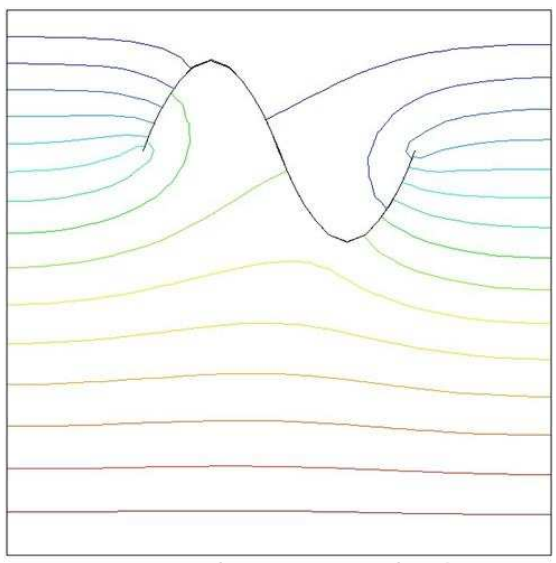

Finite Element Method

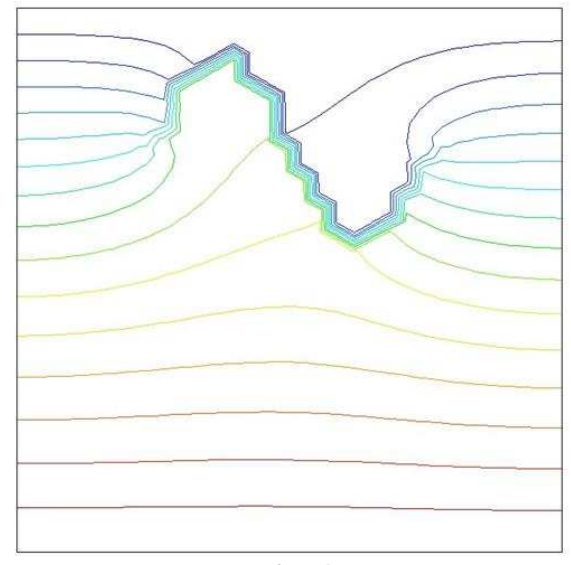

Method I

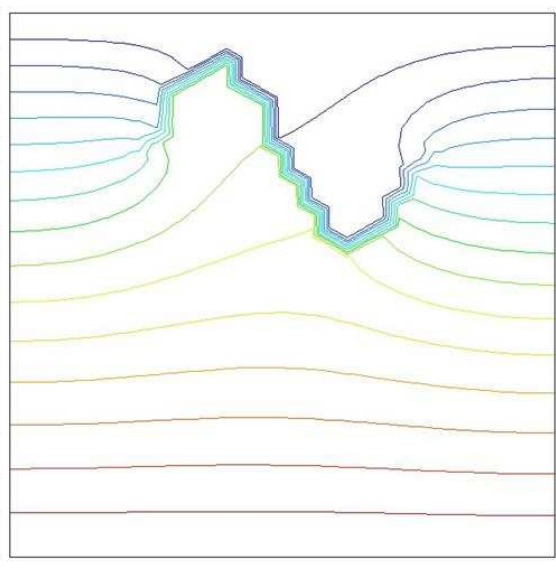

Method II

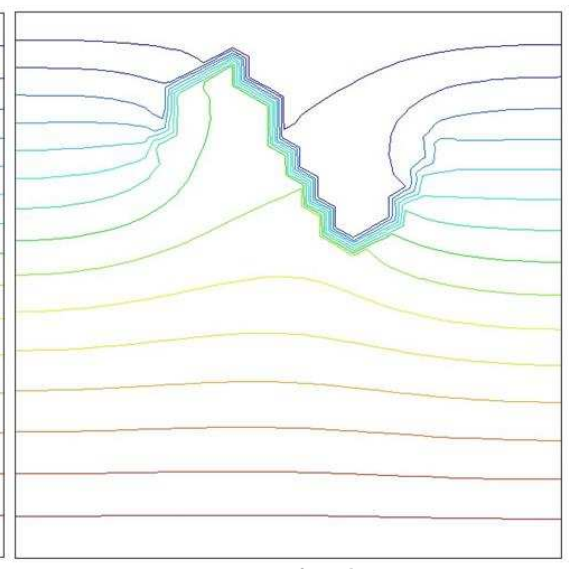

Method III

Figure 35: Temperature fields for heat conduction flow where the heat flux is set at zero $(\nabla T . n=0)$ at the interface (Mesh size equals to 0.22 ) 


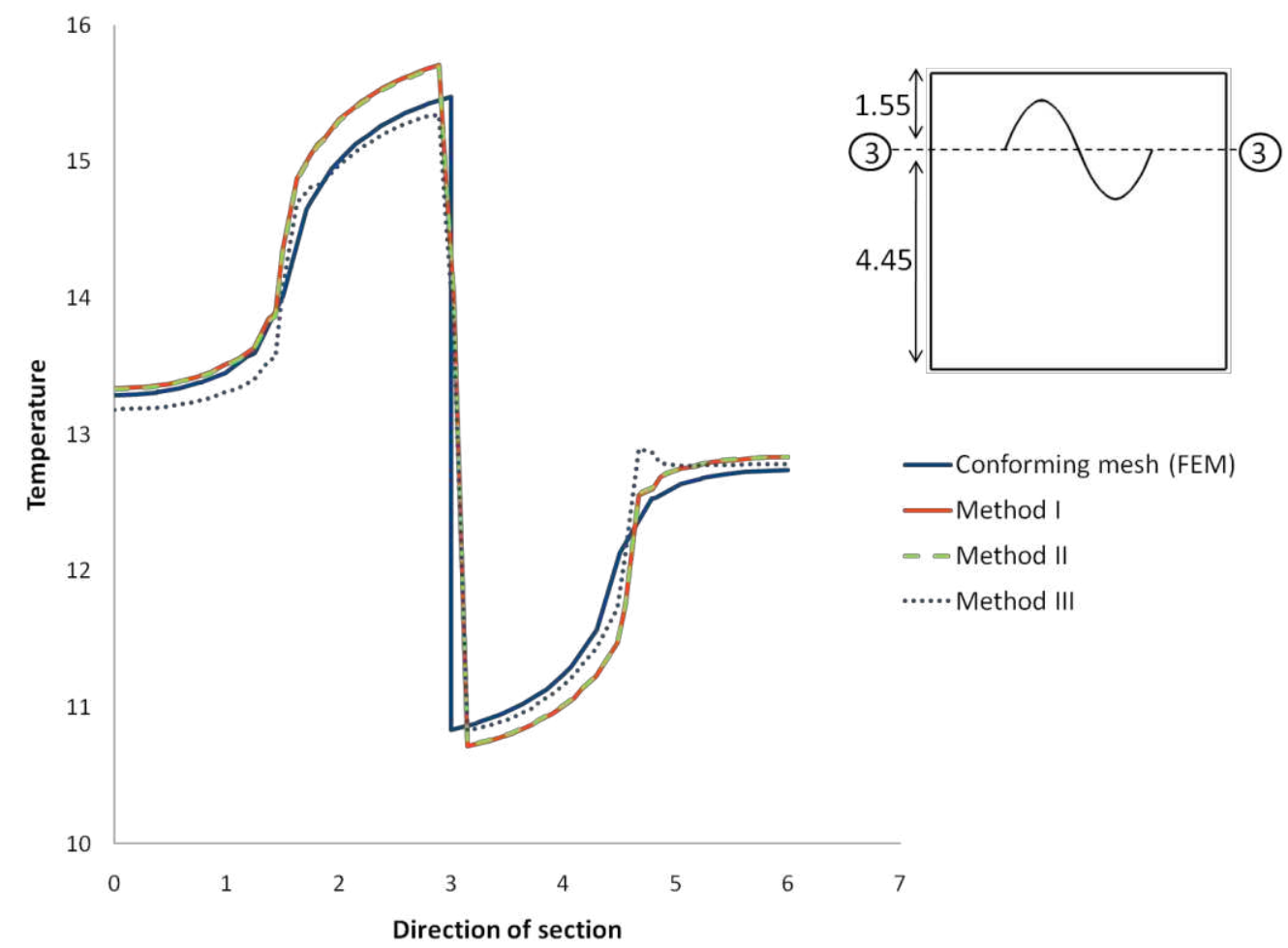

Figure 36: Comparison of the temperature field at section 3-3 where the heat flux is set at zero $(\nabla T . n=0)$ at the interface $($ Mesh size $=0.22)$

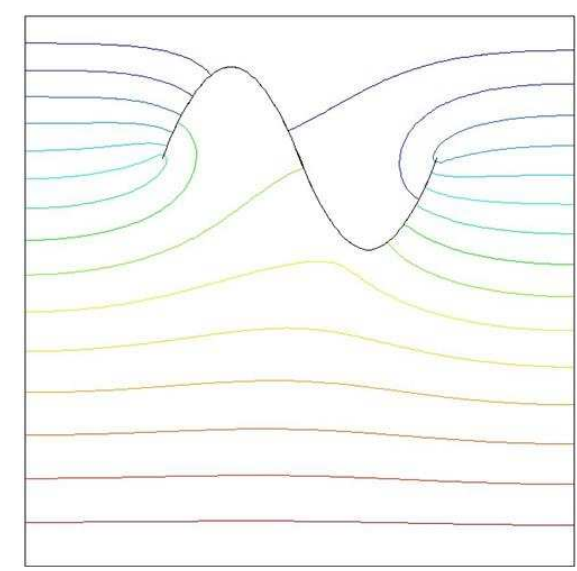

Finite Element Method

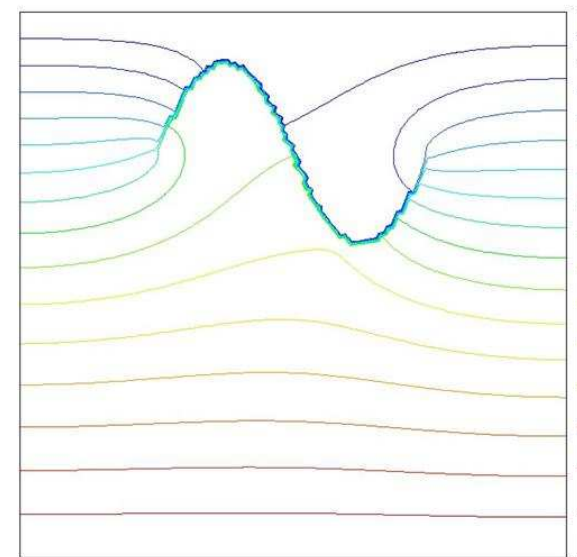

Method I



Method II

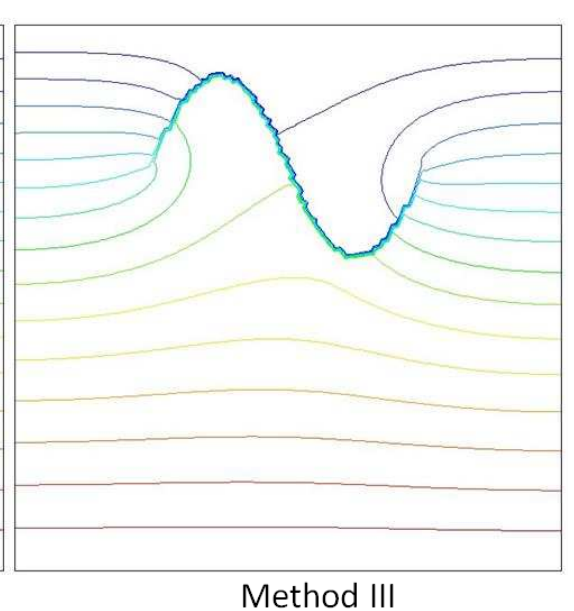

Method III

Figure 37: Temperature fields for the heat conduction flow where the heat flux is set at zero $(\nabla T . n=0)$ at the interface (Mesh size equals to 0.055 ) 

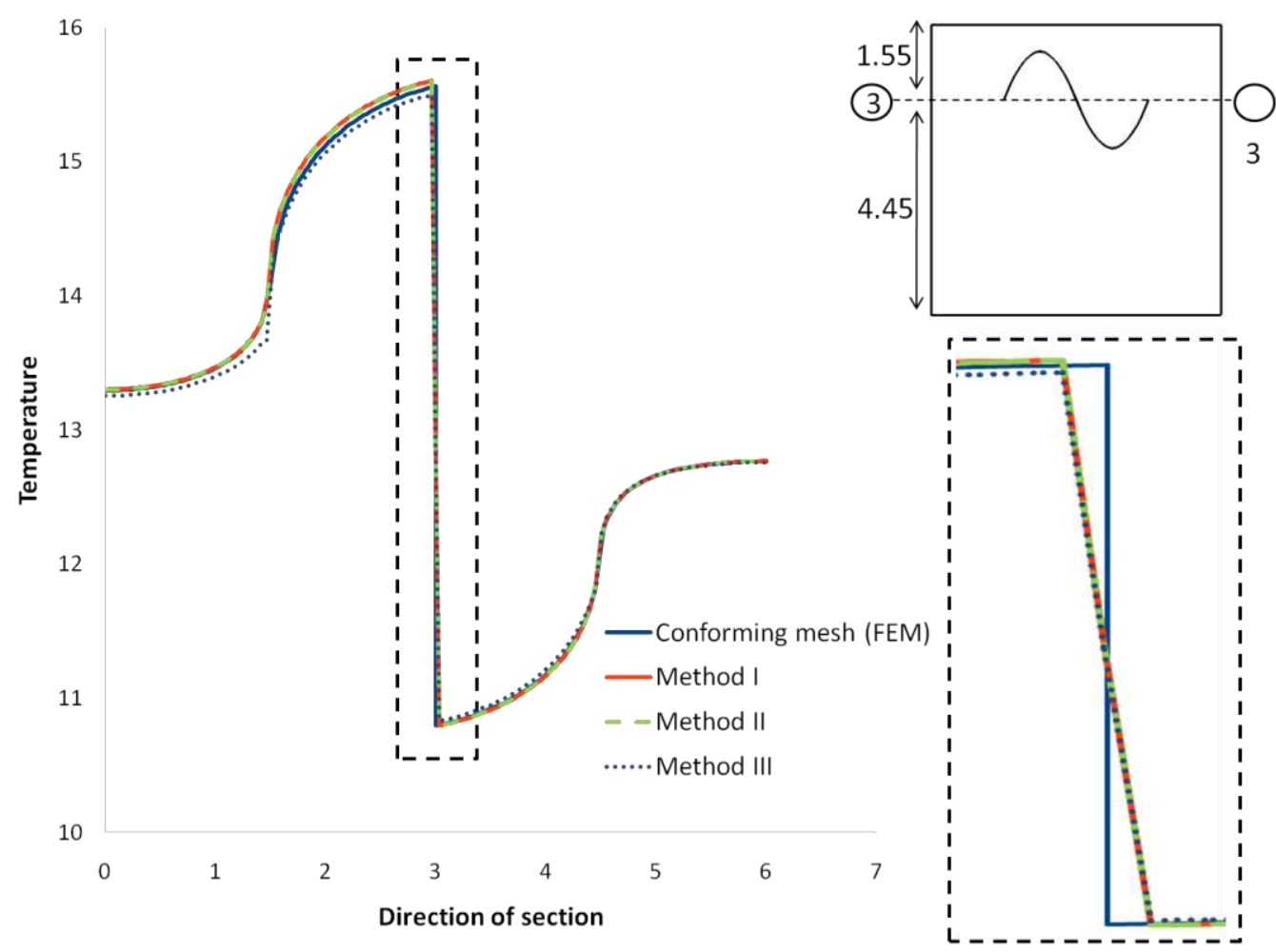

Figure 38: Comparison of the temperature field at section 3-3 for the heat conduction flow where the heat flux is set at zero $(\nabla T . \mathrm{n}=0)$ at the interface (mesh size equals to 0.055$)$

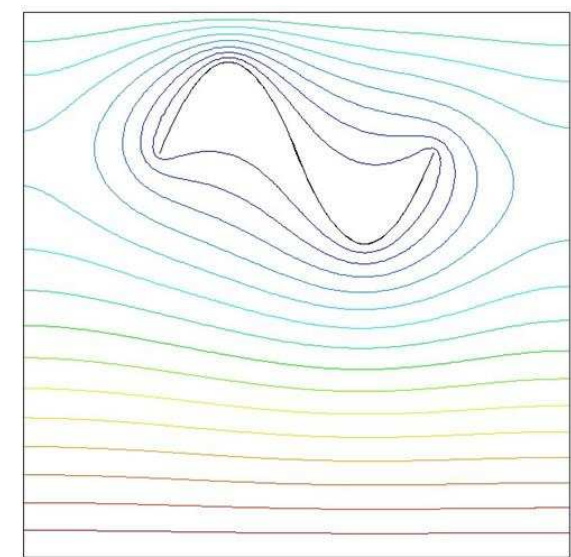

Finite Element Method
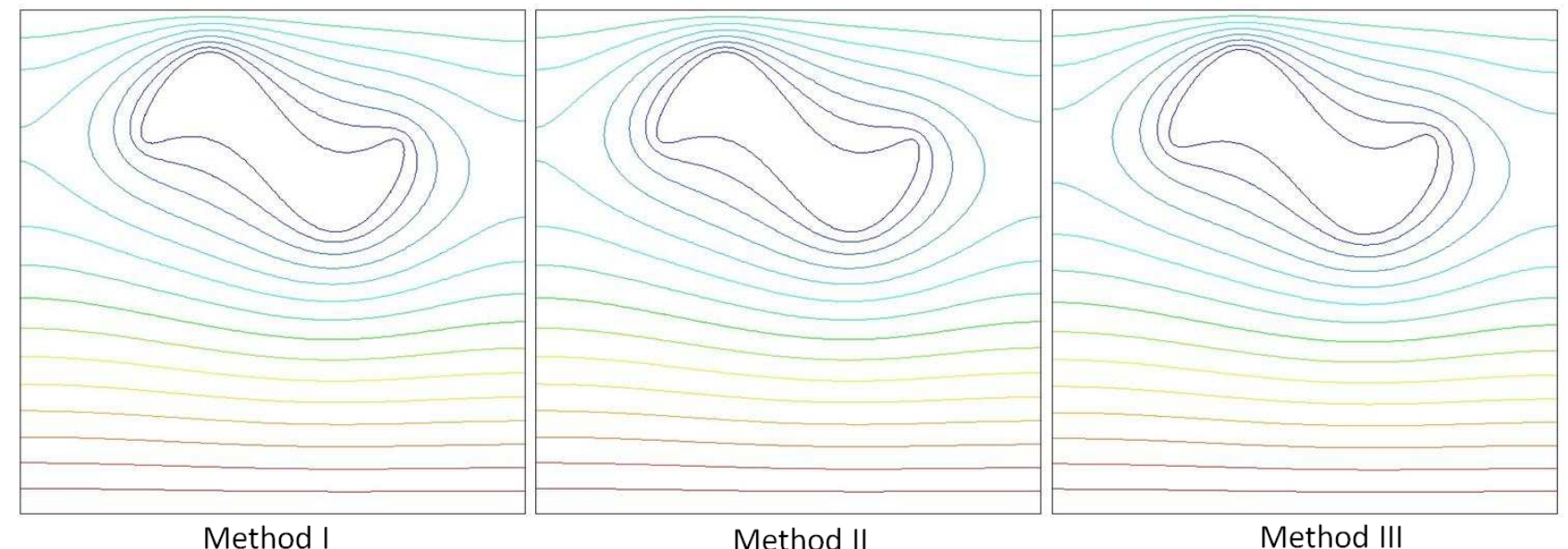

Figure 39: Temperature fields for the heat conduction flow where a zero-prescribed temperature $\left(\mathrm{T}_{\text {int }}=0\right)$ is imposed at the interface (Mesh size equals to 0.22 ) 




Figure 40: Comparison of the temperature field at section 3-3 for the heat conduction flow where a zero-prescribed temperature is imposed at the interface (Mesh size $=0.22$ )

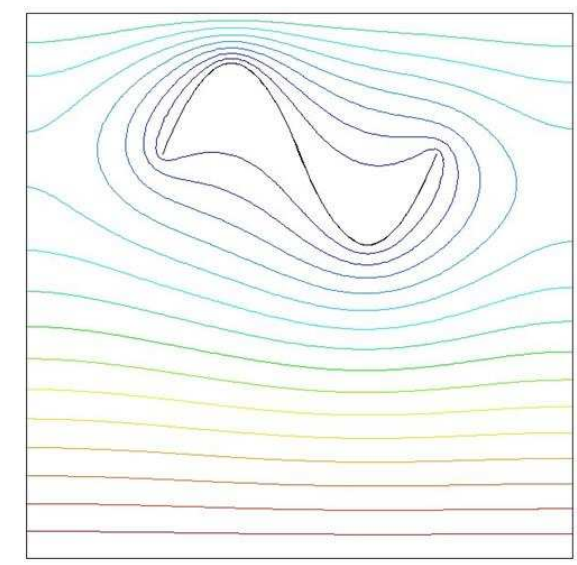

Finite Element Method

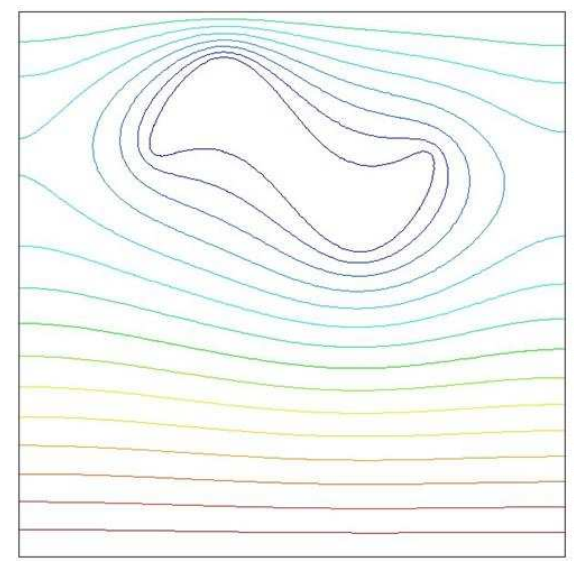

Method I

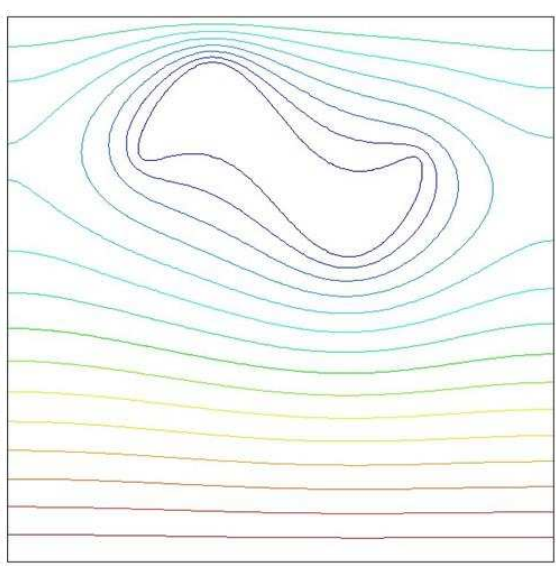

Method II

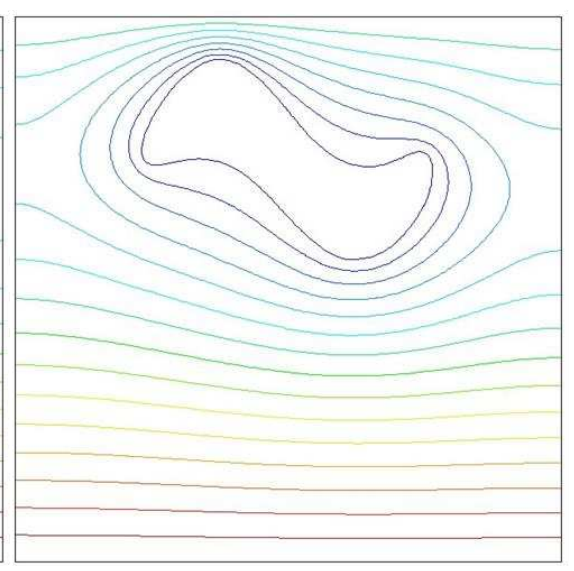

Method III

Figure 41: Temperature fields for the heat conduction flow where a zero-prescribed temperature is imposed at the interface $($ Mesh size $=0.055)$ 




Figure 42: Comparison of the temperature field at section 3-3 for the heat conduction flow where a zero-prescribed temperature is imposed at the interface $($ Mesh size $=0.055)$

\section{CONCLUSION}

Three embedded methods have been proposed for a heat conduction problem with embedded discontinuities so that aligned at arbitrary angles with respect to the mesh edges. The first one comprises a local enrichment of the FE space. This approach adds four additional degrees of freedom locally with the objective of capturing discontinuities within the element. In this case, in order to impose the Dirichlet constraint, the Lagrange multiplier method has been considered. One of the features of this method is that the system formed by the enrichment variables and the Lagrange multipliers can be statically condensed at once prior to assembly.

The second method, similar to the first one, consists of an enrichment of the FE space. This method differs from Method I due to the fact that the enriched functions are obtained by the incorporation of a characteristic user function $\phi$ with the standard nodal shape functions. Thus, three enrichment functions are used for the capturing of the discontinuity within the element (we add three new additional degrees of freedom). Here, the Lagrange multipliers and the additional degrees of freedom can be statically condensed at once before the final assembly, as well as the first method.

The third method having the ability to capture discontinuity within the element not by enrichment functions but by local modifications in the nodal shape function of the elements has also been explored. The penalty method has been chosen to impose the Dirichlet boundary condition on the interface. Therefore, the size and the graph of the final linear system are not changed. The computational cost is reduced due to the omission of the re-calculation of the matrix graph. The obvious advantage of the third method, compared to the first two cases, is that the $\mathrm{C}^{0}$ continuity can be ensured between the adjacent elements. So, finally it can be concluded that

- In all methods, the computational cost is reduced because the size and the graph of the final linear are kept the same as the standard case; 
- In the first two methods, we add some new functions whose amplitude is governed by the internal degrees of freedom that do not depend on any of the neighbouring elements;

- Although in the first two methods the $\mathrm{C}^{0}$ continuity is violated across the edges intersected by the interface, it is seen how the methods appear to work satisfactorily in real cases despite this defect; and

- All the methods show acceptable results and exhibit the potential to be suitable alternative to the other existing FE spaces with embedded discontinuities.

\section{ACKNOWLEDGEMENT}

The authors wish to acknowledge the support of the ERC through the uLites (FP7-314891), NUMEXA (FP7-611636) and REALTIME (FP7-246643) projects

1.

\section{REFERENCES}

Sven, G. and R. Arnold, An extended pressure finite element space for two-phase incompressible flows with surface tension. 2007, Academic Press Professional, Inc. p. 40-58.

2.

Sawada, T. and A. Tezuka, LLM and X-FEM based interface modeling of fluid thin structure interactions on a non-interface-fitted mesh. Computational Mechanics, 2011. 48(3): p. 319332.

3.

Motasoares, C.A., et al., An enriched space-time finite element method for fluid-structure interaction - Part I: Prescribed structural displacement, in III European Conference on Computational Mechanics. 2006, Springer Netherlands. p. 399-399.

4.

Henning, S. and F. Thomas-Peter, The extended finite element method for two-phase and free-surface flows: A systematic study. 2011, Academic Press Professional, Inc. p. 33693390.

5.

Fries, T.-P. and T. Belytschko, The extended/generalized finite element method: An overview of the method and its applications. International Journal for Numerical Methods in Engineering, 2010. 84(3): p. 253-304.

6.

Coppola-Owen, A.H. and R. Codina, Improving Eulerian two-phase flow finite element approximation with discontinuous gradient pressure shape functions. International Journal of Numerical Methods in Fluids, 2005. 49(12): p. 1287-1304.

7.

Chessa, J. and T. Belytschko, An Extended Finite Element Method for Two-Phase Fluid. journal of Applied Mechanics, 2003. 70: p. 10-17.

8 .

Belytschko, T., et al., Arbitrary discontinuities in finite elements. International Journal for Numerical Methods in Engineering, 2001. 50: p. 993-1013.

9.

O.C. Zienkiewicz and R.L. Taylor, The finite element method-the basis. 2000, Oxford,: Butterworth-Heinemann. 
10.

Chessa, J., P. Smolinski, and T. Belytschko, The extended finite element method (XFEM) for solidification problems. International Journal for Numerical Methods in Engineering, 2002. 53: p. 1959-1977.

11.

Ausas, R.F., F.S. Sousa, and G.C. Buscaglia, An improved finite element space for discontinuous pressures. Computer Methods in Applied Mechanics and Engineering, 2010. 199: p. 1019-1031.

12.

Sebastian, K. and M. Kurt, Levelset based fluid topology optimization using the extended finite element method. Structural and Multidisciplinary Optimization 2012. 46(3): p. 311 326.

13.

Rivera, C.A., et al., Parallel finite element simulations of incompressible viscous fluid flow by domain decomposition with Lagrange multipliers. Journal of Computational Physics 2010. 229(13): p. 5123-5143.

14.

Belytschko, T., Y. Lu, Y, and L. Gu, Element free galerkin methods. International Journal for Numerical Methods in Engineering, 1994. 37(2): p. 229-256.

15.

Belgacem.F.B, The mortar finite element method with lagrange multipliers. Numerische Mathematik, 1999. 84.

16.

Zhu, T. and S.N. Atluri, A modified collocation method and a penalty formulation for enforcing the essential boundary conditions in the element free Galerkin method. Comput. Mech., 1998. 21(3): p. 211-222.

17.

Hansbo.A and Hansbo.P, An unfitted finite element method, based on Nitsche's method, for elliptic interface problems. Computer Methods in Applied Mechanics and Engineering, 2002. 191: p. 5537 - 5552.

18.

Griebel, M. and M.A. Schweitzer, A particle-partition of unity method. Part V: Boundary conditions, in: S. Hildebrandt, H. Karcher (Eds.), Geometric Analysis and Nonlinear Partial Di $\square$ erential Equations. Springer, 2002: p. 517-540.

19.

Babuska, I., U. Banerjee, and J.E. Osborn, Meshless and generalized finite element methods: A survey of some major results, in: M. Griebel, M. A. Schweitzer (Eds.), Meshfree methods for partial differential equations. Lecture Notes in Computational Science and Engineering, Springer-Verlag, 2001. 26: p. 1-20. 